\title{
Flow in the exit of open pipes during acoustic resonance
}

\author{
By J. H. M. DISSELHORST AND L. VAN WIJNGAARDEN \\ Twente University of Technology, \\ Enschede, The Netherlands
}

(Received 9 July 1979 and in revised form 30 November 1979)

The flow near the mouth of an open tube is examined, experimentally and theoretically, under conditions in which resonant acoustic waves are excited in the tube at the other end. If the edge of the tube is round, separation does not occur at high Strouhal numbers, which enables us to verify theoretical predictions for dissipation in the boundary layer and for acoustic radiation. Observation with the aid of schlieren pictures shows that in the case of a sharp edge vortices are formed during inflow. The vortices are shed from the pipe during outflow. Based on these observations a mathematical model is developed for the generation and shedding of vorticity. The main result of the analysis is a boundary condition for the pressure in the wave, to be applied near the mouth. The pressure amplitudes in the acoustic wave measured under resonance are compared with theoretical predictions made with the aid of the boundary condition obtained in the paper.

\section{Introduction}

The subject of this paper is the investigation of flow phenomena in open tubes when acoustic waves are excited in these tubes near the lowest resonance frequency. In a linear non-dissipative theory, such waves reach infinite amplitudes of velocity and pressure. When viscous and thermal dissipation in the main wave and in the boundary layers along the wall are taken into account, it appears that these mechanisms keep pressures and velocities as low as observed in practice, only for very small amplitudes of excitation. Hence nonlinear effects have to be included for larger excitation amplitudes. With standing waves in closed tubes this has been done satisfactorily in the theories by Chu \& Ying (1963) and Chester (1964) which both, along different lines, describe the formation of shock waves near resonance frequencies. If the excitation, a piston for example, has a velocity of order $\delta$ and the amplitude of the resulting standing wave is of order $\epsilon$, the work done by the piston is of order $\delta \epsilon$ whereas the dissipation in the shock wave is of order $\epsilon^{3}$. From this it follows that $\epsilon \sim \delta^{\frac{1}{2}}$. This conclusion, as well as other more detailed ones from the aforementioned theories, is well confirmed by experiments, like those by Cruikshank (1972).

Nonlinearity has also been considered for propagation in open tubes. It appears that nonlinearity in the wave becomes effective only when velocity and pressure disturbances are taken into account to the third order in $\epsilon$. With closed tubes, this happens already in the second-order theory. The reason for this is essentially that a compression wave is reflected against an open end as an expansion wave. Nonlinear steepening, with an associated increase in wave velocity, gained during one traverse 
(a)

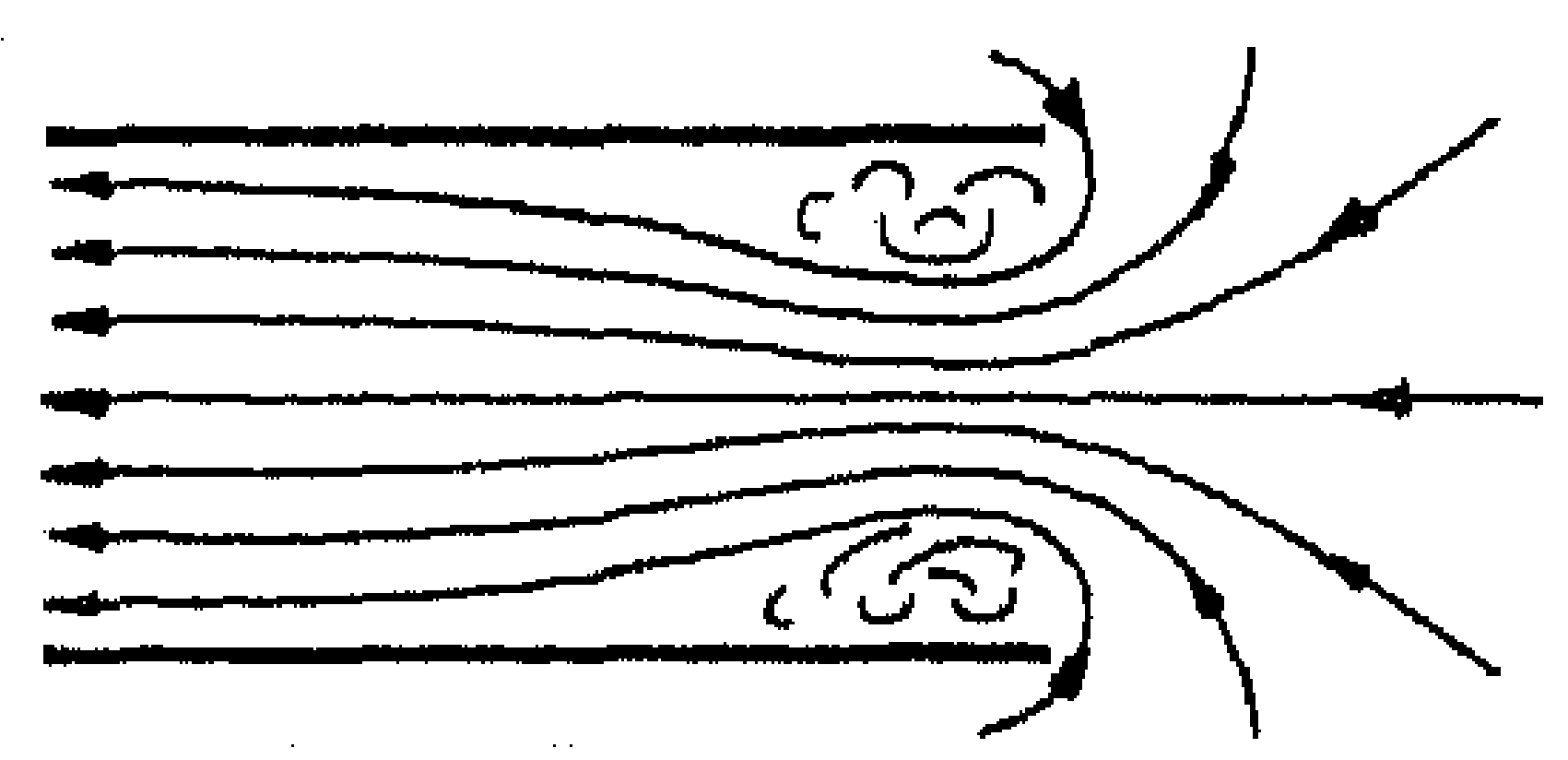

(b)

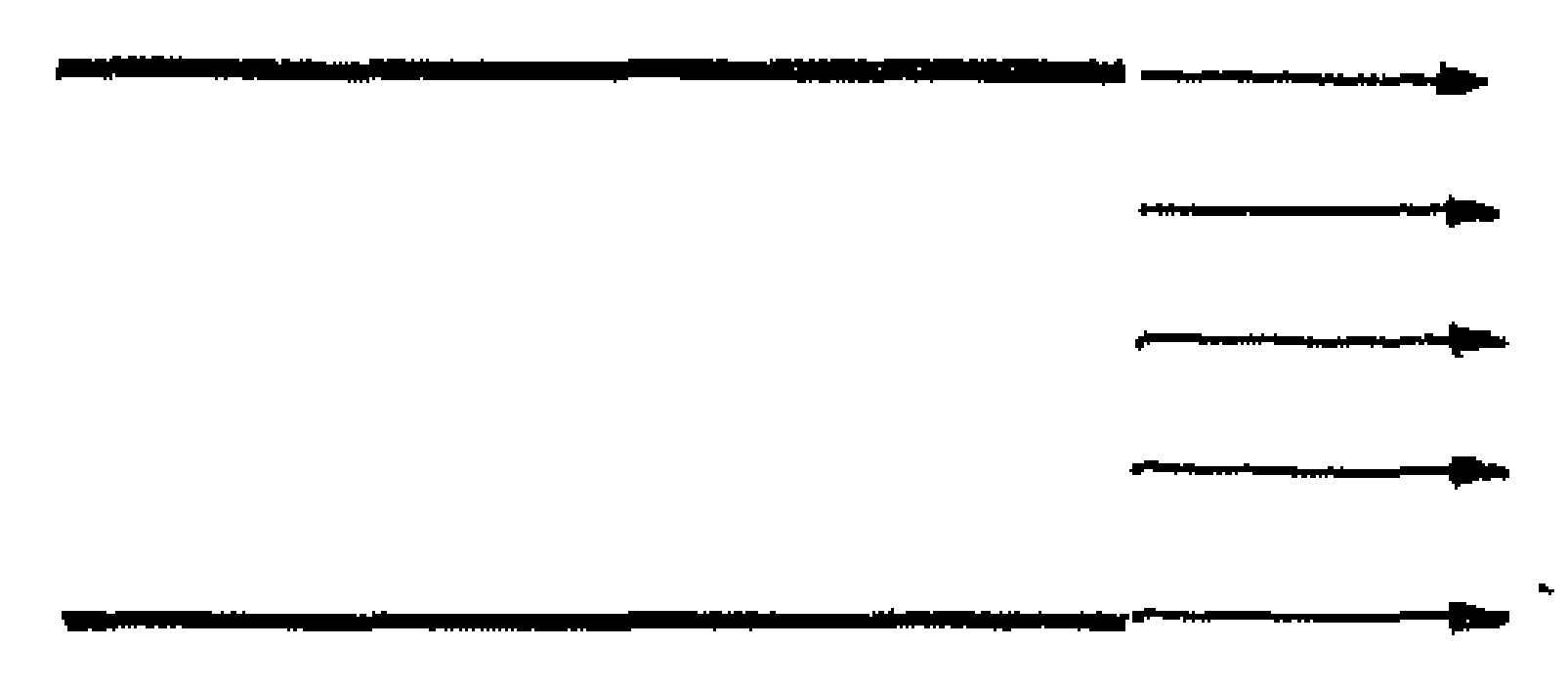

Frgure 1. Sink-like inflow $(a)$ and jet-like outflow $(b)$ for open pipe. The boundary conditions (1.1) (Van Wijngaarden 1968) are based on this flow behaviour.

of the tube is therefore almost lost during the next. Against a closed end a compression wave is reflected as a compression wave again. The outcome of third-order theories (Seymour \& Mortell 1973; Jimenez 1973) is that $\epsilon \sim \delta^{\frac{1}{3}}$, a result which is not confirmed by the bulk of experimental results such as those of Sturtevant (1974). For sharpedged open pipes Van Wijngaarden (1968) drew attention to jet formation at the mouth during outflow and boundary-layer separation during inflow as possible dissipative mechanisms. Based on the flow behaviour sketched in figure 1, he proposed as the condition for the pressure disturbance $p-p_{0}$ at the mouth of the pipe

$$
\begin{aligned}
& p-p_{0}=0 \text { at outflow } \\
& p-p_{0}=c \frac{\partial u}{\partial t}-\rho_{0} u^{2} \quad \text { at inflow }
\end{aligned}
$$

where $\rho_{0}$ and $p_{0}$ are the ambient atmospheric density and pressure, $u$ denotes the velocity, $t$ denotes time and $c$ is a constant. A theory along the lines of Chu \& Ying (1963) has been given in Van Wijngaarden (1968) in which (1.1) is imposed at the open end of the tube. In retrospect this theory could have been much simpler, as pointed out later by Seymour \& Mortell (1973), when it was realized that nonlinear effects in the main wave appear only in third-order theory. In the case when boundarylayer separation dominates we have to deal with a second-order problem. It is known from experiments (Lettar 1939; Sturtevant 1974) that with very steep pressure signals shock waves also appear in the open tube case, showing that eventually nonlinear distortion plays an important part. Yet the situation treated here, where the boundary condition at the open end determines the flow, is far from clear. In Van Wijngaarden (1968) viscosity and thermal conduction are neglected.

Further experiments reported in Van Wijngaarden \& Wormgoor (1974) show that the picture in figure 1 is too simple. It would do if the flow were quasi-steady. In general, however, vortices are generated during inflow and expelled during outflow. This unsteady process affects the standing wave in the tube significantly. We decided therefore to make more detailed observations of the flow behaviour near the mouth and to attempt to explain these observations quantitatively.

To assess the role of viscous and thermal dissipation in boundary layers along the wall we also considered tubes with round edges for which, at least at low amplitudes, there is no boundary-layer separation at inflow or outflow. This type of standing wave, entirely determined by dissipation and by acoustic radiation from the end, is discussed in $\S 2$. In $\S 3$ the experimental set-up is briefly described. In $\S 4$ results of the experi- 
ments are given in particular for aspects which concern vortex generation and vortex trajectories near the open end. A mathematical model which is based on these observations is given in $\S 5$. Since for practical applications the main interest is in formulating a boundary condition at the open end for the standing wave, the model is constructed so as to enable the calculation of the difference between the pressure in the wave near the mouth and the ambient pressure. In $\S 6$ the numerical scheme is described, with the help of which the rolling up of vortex sheets coming from the sharp edges is calculated numerically. Numerical results for the trajectories of vortices and for pressures near the mouth of an open pipe are given in $\$ 7$.

From the numerical results a boundary condition for the pressure at the open end follows. A refinement is that this should be applied not at the end but at a section at $0.61 R$ ( $R$ being the tube's radius) outward in the axial direction. This is implied by the results of Levine \& Schwinger (1948) who found that radiation of sound from an unflanged pipe can be allowed for by lengthening the pipe by an amount $0.61 R$ (at large wavelengths) in the calculation, thereby imposing ambient pressure at this (fictitious) open end. In the new boundary condition the time dependence of the pressure $p-p_{0}$ is the same as that of the velocity $u$, and in amplitude $p-p_{0} \sim u^{5}$, a consequence of potential theory. The work done by the gas leaving the pipe on the ambient atmosphere follows from the proposed boundary condition. The mean energy balance of the gas in the pipe states that this and the power dissipated by heat conduction and viscosity together with the radiated acoustic power balance the work done on the gas by the driving piston. The latter can easily be measured and in this way a verification of the proposed boundary condition is possible.

The comparison between predicted and measured results is made at the end of $\S 7$ in terms of the three dimensionless parameters by which the physical mechanisms can be characterized. They are listed here. The angular frequency $\Omega$, tube radius $R$ and viscosity $\nu$ of the medium, in our case air, define a dimensionless number representing the ratio between the thickness $(\nu / \Omega)^{\frac{1}{2}}$ of the boundary layer at the wall and the tube radius $R$. It is denoted here by the abbreviation $S h$, standing for 'shear',

$$
S h=\left(\nu / \Omega R^{2}\right)^{\frac{1}{2}} .
$$

Then there is the ratio between local acceleration and convective acceleration. A measure for this is the Strouhal number $S t$. If the maximum velocity during a cycle is $\hat{u}$, the pertinent Strouhal number here is

$$
S t=\Omega R / \hat{\imath} .
$$

For large frequencies and small velocity amplitudes $S t$ is large, and vice versa. Finally, acoustic radiation is characterized by the ratio between tube radius and wavelength. With velocity of sound $a_{0}$ in the ambient atmosphere, this ratio, denoted here by $K$, is

$$
K=\Omega R / a_{0}
$$

At the resonance frequencies at which our experiments are done

$$
K=\pi R / 2 L .
$$

In our experiments $K$ is always a small number varying between 0.01 and 0.08 . The parameters $S h, S t$ and $K$ are measures of the principal mechanisms, viscous action, nonlinearity and radiation, which we shall consider. 


\section{Waves in tubes with a round edge}

Standing waves in tubes with a round edge are primarily investigated here because no boundary-layer separation occurs, at least at low amplitudes of the excitation. The external work on the gas in the tube is partly dissipated in the boundary layer along the wall and partly radiated from the open end as sound. These can both be predicted accurately with, for the dissipation, the restriction of laminar flow in the boundary layer. Merkli (1973), in his extensive experimental study of acoustic oscillations in tubes, finds that turbulent flow in the boundary layer occurs when $\hat{u} /(\Omega \nu)^{\frac{1}{2}}$ exceeds a value of 350-700. In terms of the dimensionless parameters used here this means that laminar flow in the boundary layer may be assumed as long as $(S t S h)^{-1}<500$, say, a condition which is satisfied in most of the experiments discussed later in this paper. A theory of boundary layers in oscillating flows has been given in Rayleigh (1945). 'Temkin (1968) finds for the dissipation $P_{w}$ in an oscillating pipe flow, with Prandtl number $\operatorname{Pr}$, ratio of specific heats $\gamma$,

$$
P_{w}=\frac{1}{2} \rho_{0} \hat{u}^{2}\left(\frac{1}{2} \Omega \nu\right)^{\frac{1}{2}} \pi R^{2}\left(1+(\gamma-1) / P r^{\frac{1}{2}}\right) L / R .
$$

This comprises the power dissipated by viscosity and by heat conduction. The subscript $w$ indicates wall friction. An additional, though small, contribution occurs in the (smooth) flow around the edge. This has been calculated elsewhere (Disselhorst \& Van Wijngaarden 1979) and is omitted here. We cast (2.1) in a slightly different form. Since we are dealing with resonance conditions we may eliminate $L$ by using the relation, valid at the lowest resonance frequency,

$$
L=\pi a_{0} / 2 \Omega \text {. }
$$

Further, in the linear acoustic approximation there exists between $\hat{u}$ and the maximum pressure at the driving piston $\hat{p}_{p}$ the relation

$$
\hat{p}_{p}-p_{0}=\rho_{0} a_{0} \hat{u} \text {. }
$$

In an energy balance consisting of second-order terms in which we shall use (2.1), we may use (2.3). Then we find, $S h$ being defined in (1.2),

$$
P_{w}=\frac{\pi^{2}}{2^{5}} \operatorname{sh} R^{2} \frac{\left(\hat{p}_{p}-p_{0}\right)^{2}}{\rho_{0} a_{0}}\left(1+\frac{\gamma-1}{P r^{\frac{1}{2}}}\right) .
$$

The radiated acoustic power could be obtained from the results in the classic paper by Levine \& Schwinger (1948), dealing with sound radiation from an unflanged pipe. For small values of $K$, defined by (1.4) and (1.5), the radiated power can more easily be found as follows. The mass flow from the mouth is

$$
m=\rho_{0} \pi R^{2} \hat{u} e^{i \Omega t} \text {. }
$$

In the far field the mouth can be considered as the site of an acoustic monopole with strength $m$. Using the expression (see, for example, Lighthill 1978, p. 22)

$$
P=\dot{m}^{2} / 4 \pi / \rho_{0} a_{0}
$$

for the power radiated from a monopole, and adjusting for the proximity of a rigid wall at distance $d$ from the centre of the mouth using the factor

$$
\kappa=1+\frac{\sin 2 K d / R}{2 K d / R}=1+\frac{\sin \pi d / L}{\pi d / L} \text { at resonance, }
$$


we find for the radiated acoustic power $P_{\mathrm{ac}}$, averaged over a cycle,

$$
P_{\mathrm{ac}}=\kappa \frac{K^{2} \pi R^{2}\left(\hat{p}_{p}-p_{0}\right)^{2}}{8 \rho_{0} a_{0}}
$$

In resonant conditions the pressure at the piston

$$
p_{p}-p_{0}=\left(\widehat{p}_{p}-p_{0}\right) e^{i \Omega t}
$$

is in phase $\uparrow$ with the velocity of the piston when this is harmonic in time. We represent the velocity of the piston here with the real part of $\Omega \delta e^{i \Omega t}$. Then the work done by the piston over a period of the motion of the piston is

$$
P_{p}=\frac{1}{2} \Omega \delta\left(\hat{p}_{p}-p_{0}\right) \pi R^{2}
$$

yielding the energy balance

$$
P_{p}=P_{w}+P_{\mathrm{ac}}
$$

The resulting pressure amplitude of the wave motion, at given $\Omega$ and $\delta$, can be expressed in terms of the dimensionless quantity $Q$, defined as

$$
Q=\frac{\left(\hat{p}_{p}-p_{0}\right) a_{0}}{\gamma p_{0} \Omega \delta}
$$

Using this definition and the expressions $(2.4),(2.6)$ and $(2.8)$, we obtain from the energy balance (2.9)

$$
Q=\left\{\frac{\pi}{2 \sqrt{ } 2}\left(1+\frac{\gamma-1}{\operatorname{Pr} \frac{1}{2}}\right) S h+\frac{\kappa K^{2}}{4}\right\}^{-1} .
$$

Since we may use the relation (2.3) and the definition (1.3) of the Strouhal number to write $Q$ as

$$
Q=R / S t \delta
$$

an alternative expression for $(2.11)$ is

$$
S t=\left(\frac{\delta}{R}\right)^{-1}\left\{\frac{\pi}{2 \sqrt{ } 2}\left(1+\frac{\gamma-1}{P r^{\frac{1}{2}}}\right) S h+\frac{\kappa K^{2}}{4}\right\} .
$$

The relation (2.11) may be expected to hold for high values of $S t$; more precisely, when no boundary-layer separation occurs.

At large values of $\hat{u}$, corresponding to low values of $S t$, the boundary layer separates from the wall at outflow. In the limit of very low $s t$ the flow near the exit will look like that sketched in figure 2. During inflow, when owing to the round edge no separation takes plice, Bernoulli's theorem gives in the exit

$$
p-p_{0}=-\frac{1}{2} \rho_{0} u^{2}
$$

During outflow jet formation occurs and the pressure approximately equals the ambient pressure

$$
p=p_{0}
$$

$\dagger$ Of course there is a small phase lag due to viscosity and thermal conduction. This leads for small enough $S h$ to a negligible contribution to $Q$ in $(2.11)$. 
(a)

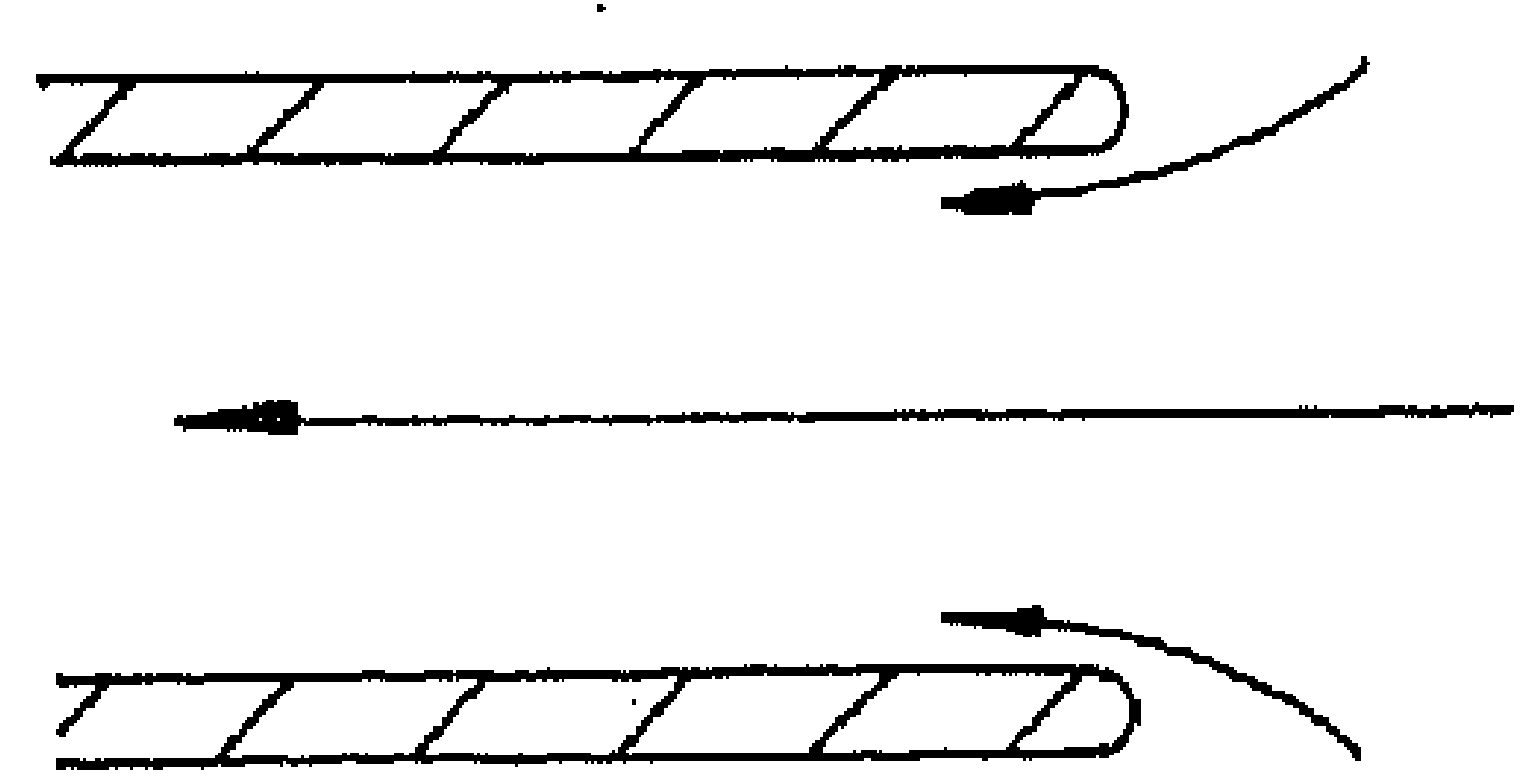

(b)

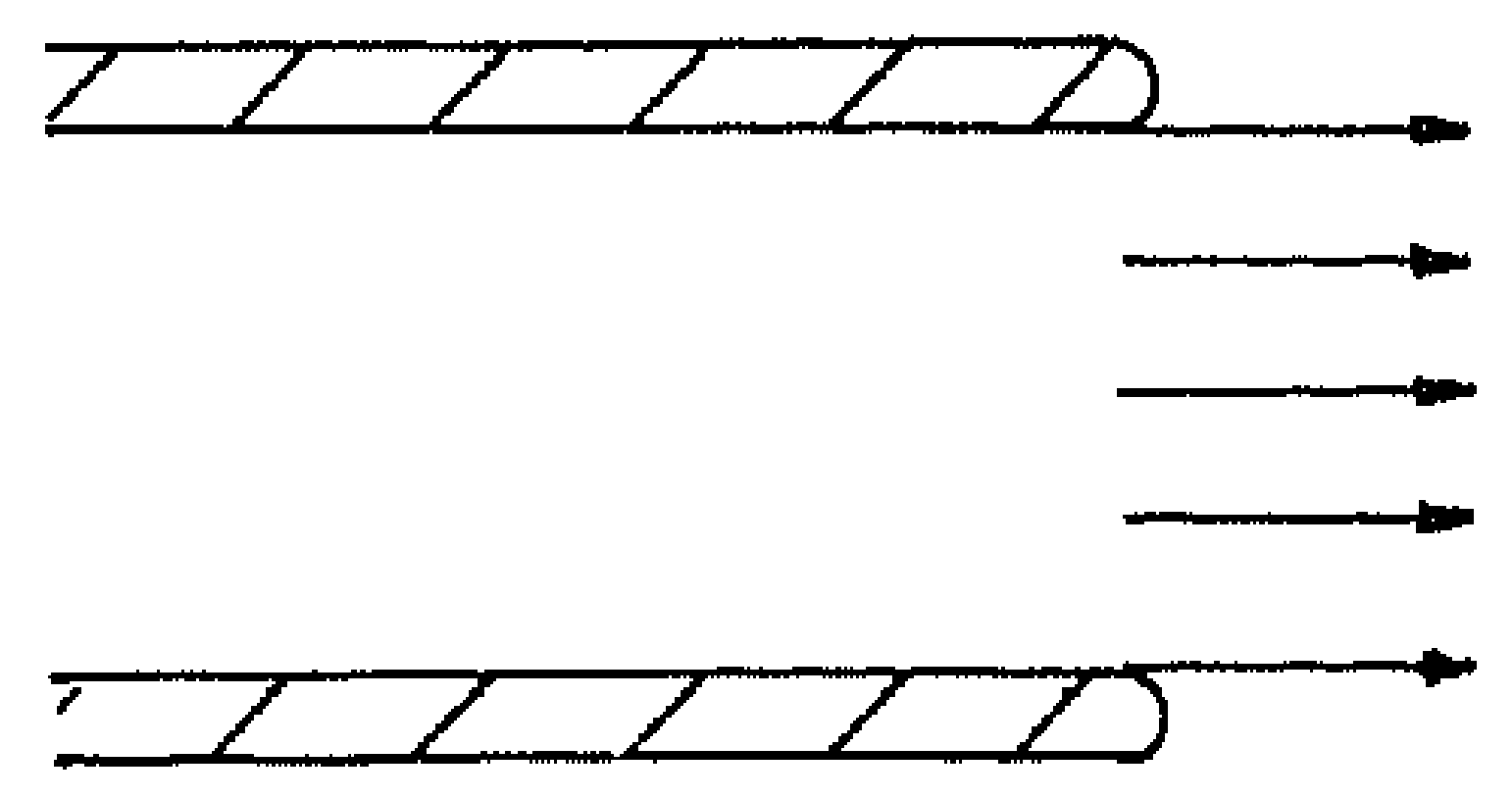

Fravre 2. Quasi-steady flow in the exit of a pipe with a round edge. With inflow $(a)$ Bernoulli's law $(2.14)$ holds; at outflow $(b)$ the flow is jet-like due to boundary-layer separation.

With sharp edges the conditions (1.1) reduce for $S t \rightarrow 0$ to

$$
\begin{aligned}
& p-p_{0}=-\rho_{0} u^{2} \text { at inflow, } \\
& p=p_{0} \text { at outflow. }
\end{aligned}
$$

The difference between the right-hand sides of $(2.14)$ and $(2.16)$ is associated with separation at inflow with the sharp edge and smooth flow with the round edge. The result of calculations of the pressure in a standing resonant wave subject to $(2.16)$ at the exit is, as given in Van Wijngaarden (1968),

$$
\frac{p_{p}-p_{0}}{\gamma p_{0}}=\left(\frac{\pi \delta}{L}\right)^{\frac{1}{2}}|\sin \Omega t|^{\frac{1}{2}} \operatorname{sgn}(\sin \Omega t) \text {. }
$$

For a harmonic $p_{p}-p_{0}$ we defined $\hat{p}_{p}-p_{0}$ as the maximum value. For a function like the above which comprises higher harmonics as well, we define $\hat{p}_{p}-p_{0}$ as $2^{\frac{1}{2}}$ times the root mean square of $p_{p}-p_{0}$ (which is, for a purely harmonic function, the maximum value). This gives for (2.17)

$$
\frac{\hat{p}_{p}-p_{0}}{\gamma p_{0}}=2\left(\frac{\delta}{L}\right)^{\frac{1}{2}}
$$

The corresponding value of $Q$ is, using (2.10), found to be

$$
Q=\frac{4}{\pi}\left(\frac{L}{\delta}\right)^{\frac{1}{2}}
$$

Likewise, in the limit of small values of $S t$ for a round-edged tube, where (2.14) holds, we obtain

$$
Q=\frac{4 \sqrt{ } 2}{\pi}\left(\frac{L}{\delta}\right)^{\frac{1}{2}}
$$

Experiments to be described in the next section were carried out with tubes both with round and with sharp edges. At resonance the pressure at the piston was measured and the experimentally obtained values of $Q$, defined in (2.10), plotted for a number of values of $L$ against $\delta / R$. The results are shown in figure 3 . We discuss first the results for pipes with a round edge. For each length $L, K$ and $S h$ are constant when $\delta$ varies because they are determined by the resonance frequency $\Omega$. For increasing $\delta$, st decreases because $\hat{u}$ increases. The broken line on the left-hand side indicates, for each $L$, the value of $Q$ predicted by (2.11) whereas that on the right-hand side indicates the relation $(2.20)$ predicted by the theory for low $S t$. The measurements clearly show 
(a)

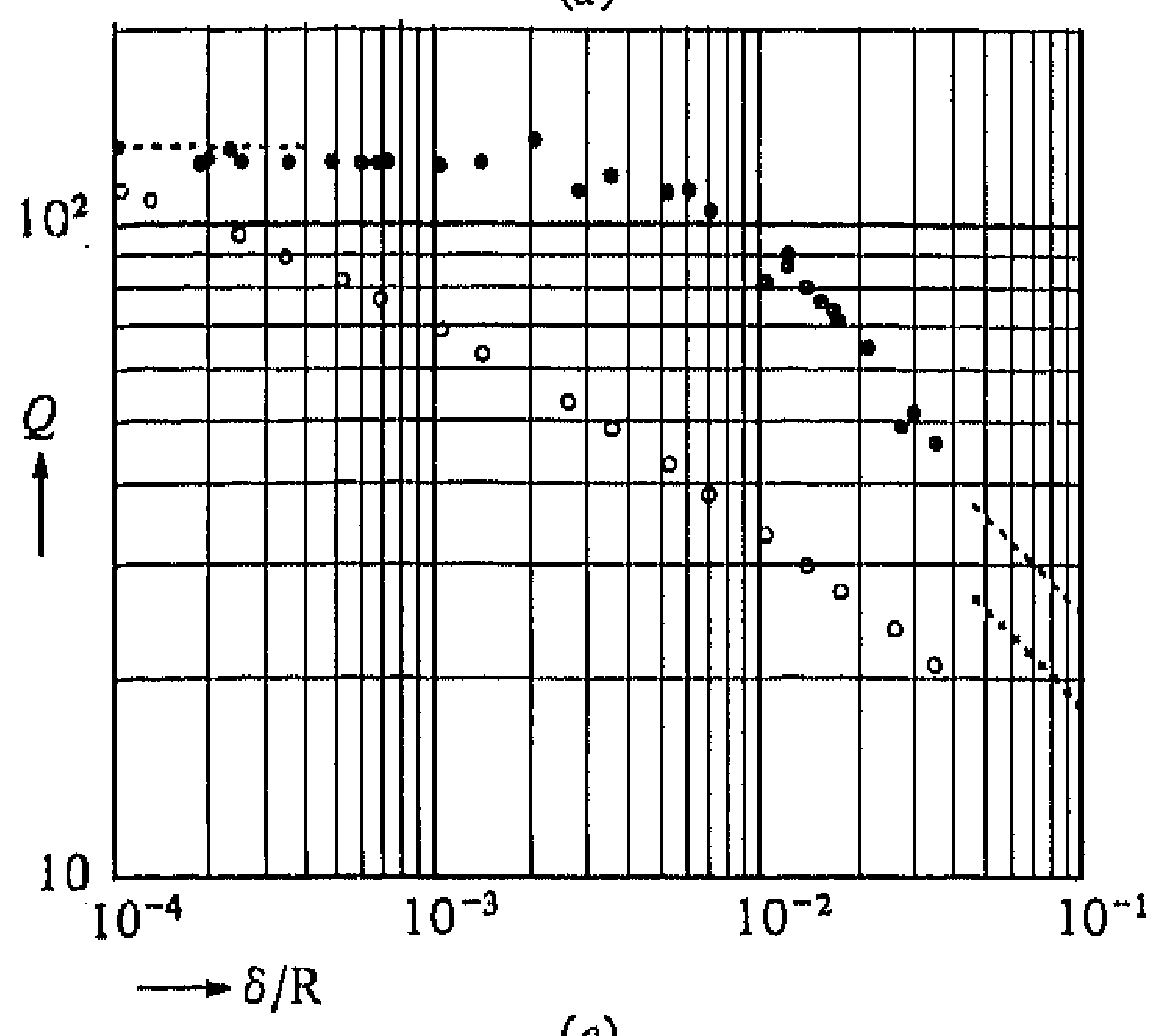

(c)

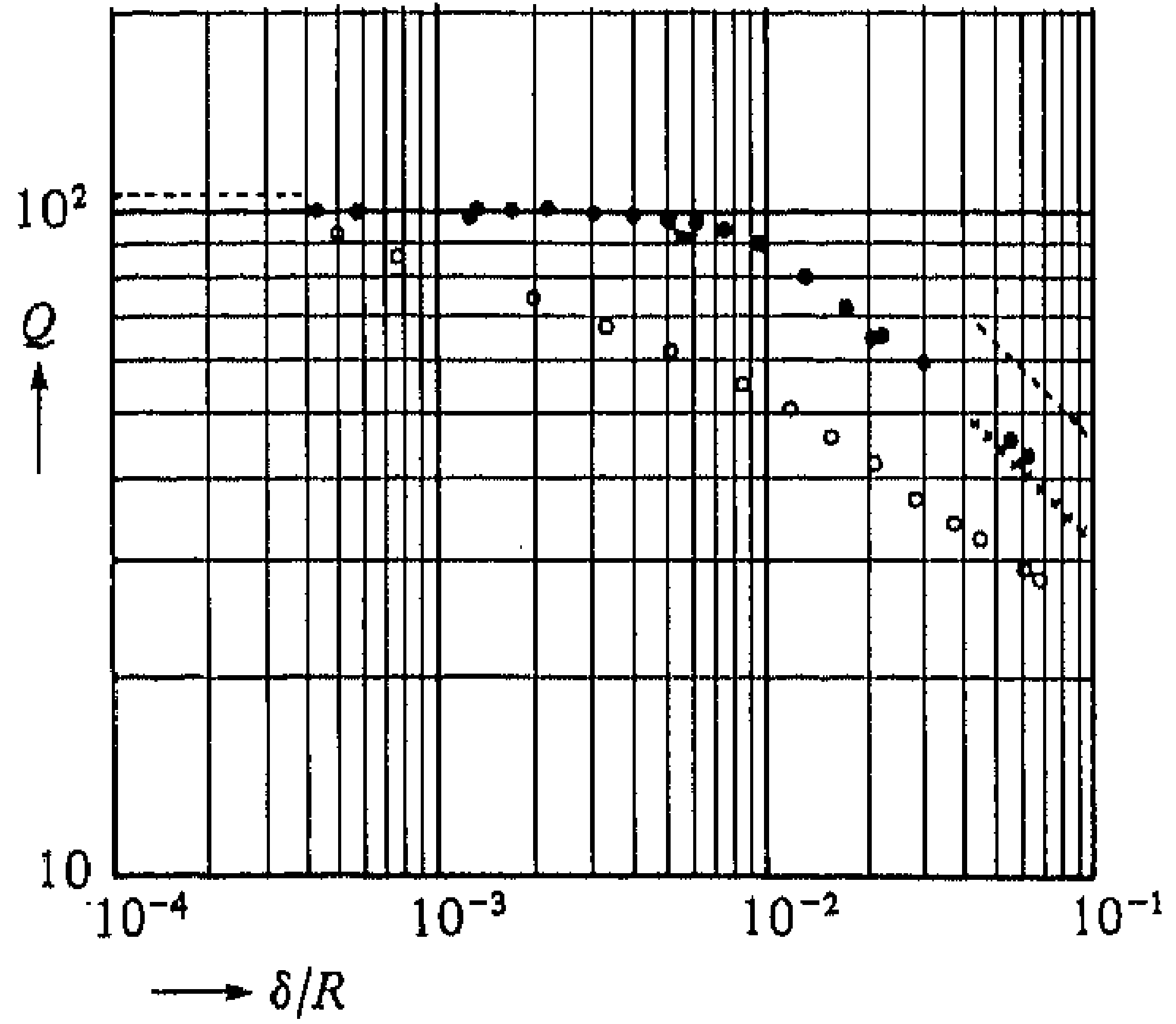

(b)

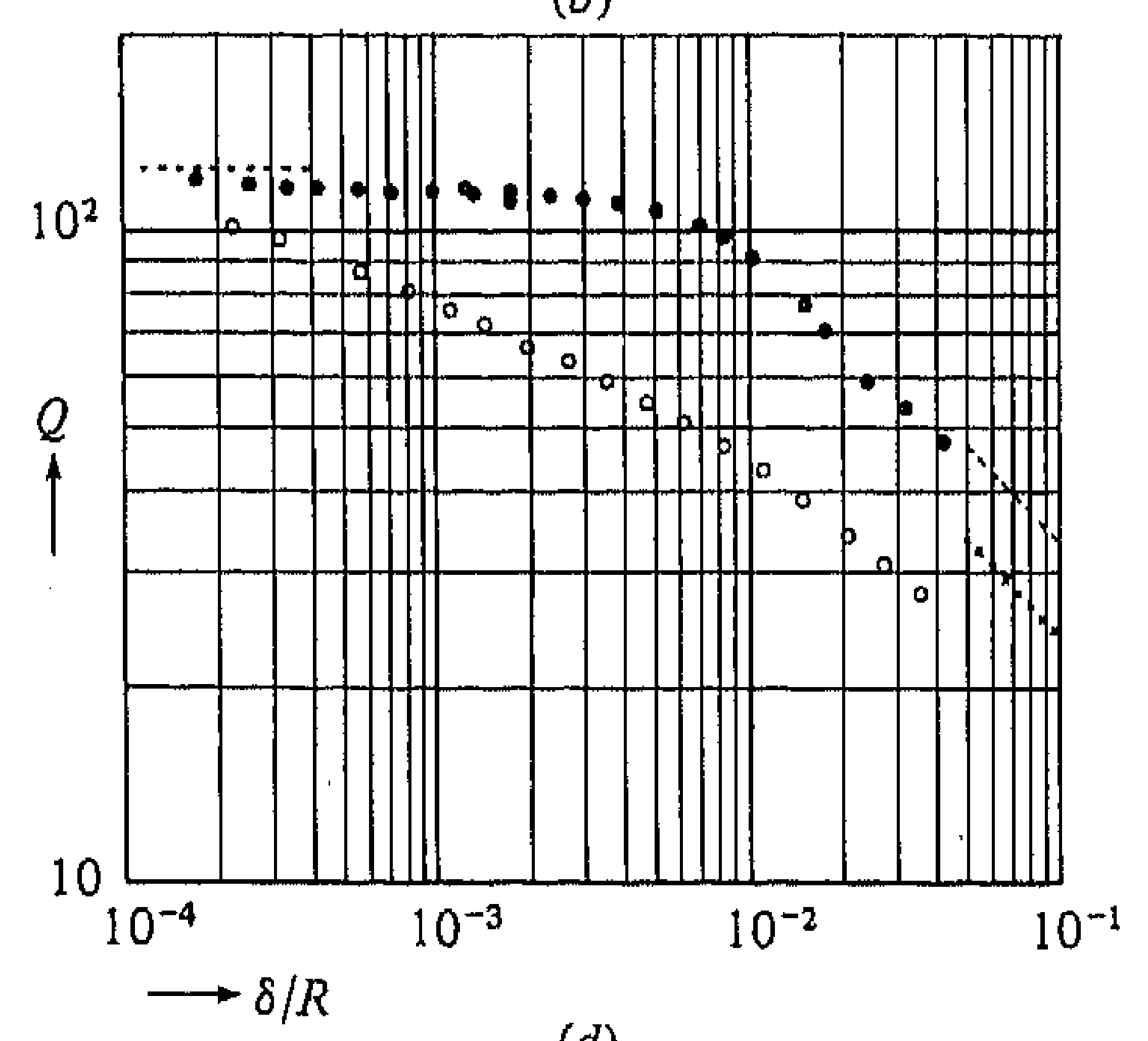

(d)

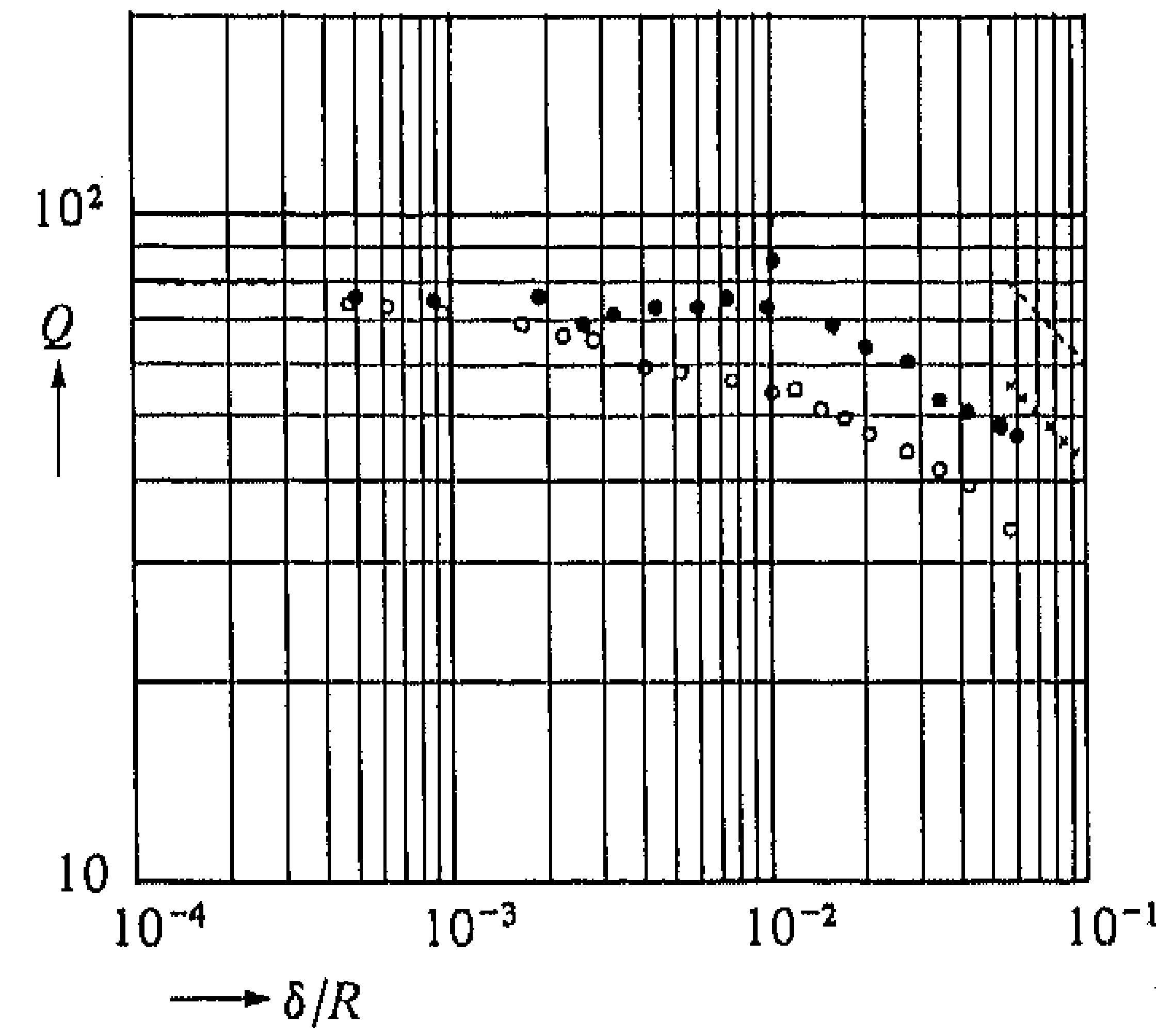

Frgure 3. The quantity $Q$ as a function of the dimensionless piston amplitude $\delta / R$ for different lengths of the tube. (a) $L=1.1 \mathrm{~m} ;(b) L=1.8 \mathrm{~m}$; (c) $L=3.3 \mathrm{~m}$; (d) $L=6.3 \mathrm{~m}$. Measurements: $O$, sharp edge; $\odot$, round edge. Theory: $x \times x$, sharp edge, equation $(2.19) ;-\cdots$, round edge, equations (2.11) and (2.20).

two regions. First, for $\delta / R$ smaller than $10^{-2} Q$ is independent of $\delta \dagger$ and very close to the theoretical value in (2.11) based upon boundary-layer dissipation and sound radiation. For $\delta / R$ larger than about $10^{-2}, Q$ decreases with $\delta$ in a way which is for larger $\delta$ (small $S t$ ) in good agreement with (2.20). From the agreement between theory and experiment at low values of $\delta / R$, we may conclude that losses due to boundary-layer dissipation and acoustic radiation are accurately described by the relation for $P_{w}$ and $P_{\mathrm{ac}}$ in (2.4) and (2.6), whence we shall use these expressions with sharp edges to separate radiation and boundary-layer dissipation from energy losses of other nature.

For tubes with a sharp edge the results in figure 3 show that there are, in the range of experimental parameters, no $\delta$ values where $Q$ is independent of $\delta / R$. At low values of $\delta / R$ (high $S t$ ) the experimental results fit neither with (2.11) (the measurements suggest, however, that for very small $\delta / R$, less than $10^{-4}$ say, end losses become negligible) nor with (2.19). At large $\delta / R$ (low $S t$ ) a tendency to approach the asymptotic

$\dagger$ More precisely, $Q$ does not depend on $\delta$ for $S t$ larger than about 1, see figure 20. For $Q \sim 10^{2}$ this amounts to [cf. (2.12)] $\delta / R \sim 10^{-2}$. 


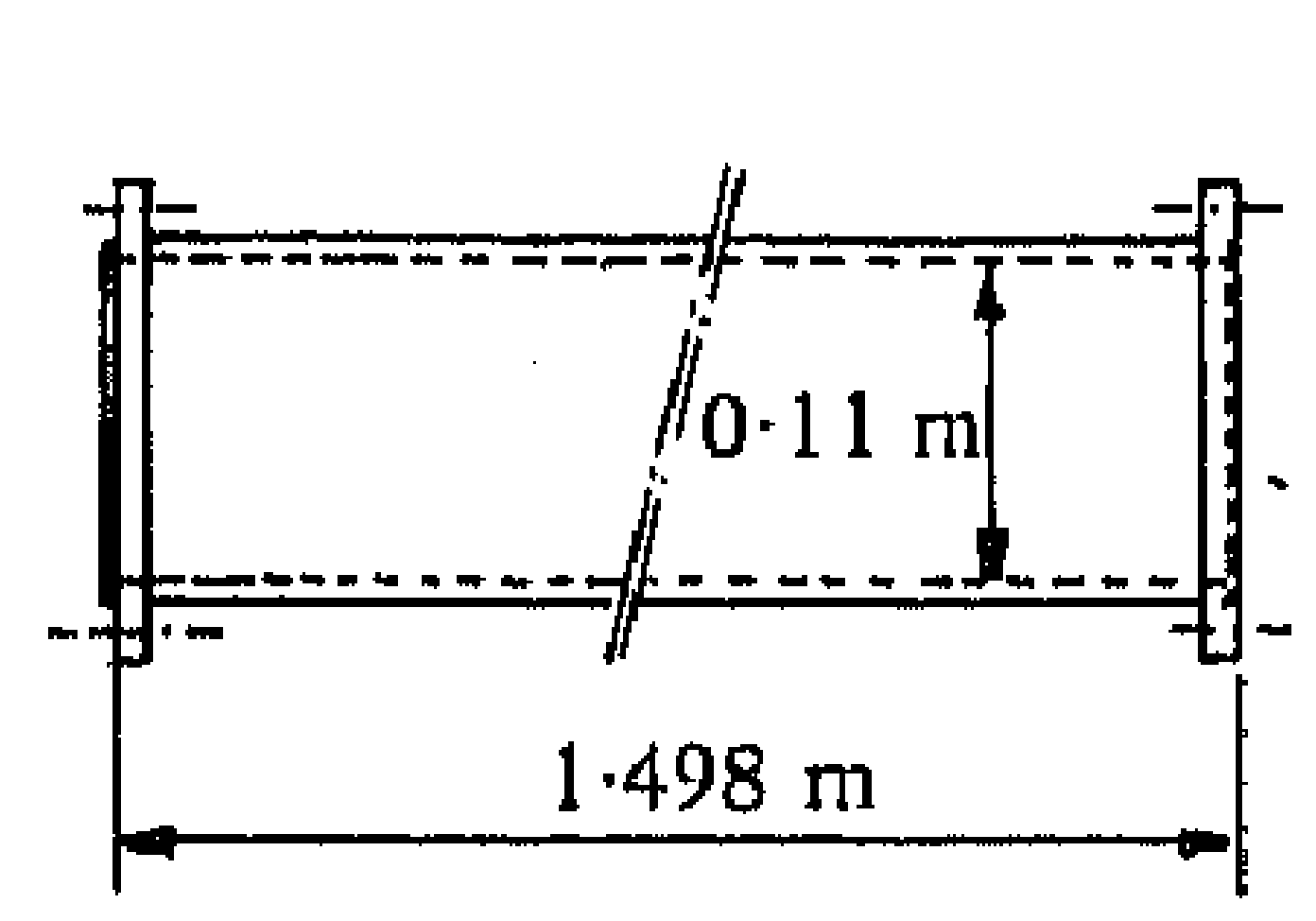

(b)

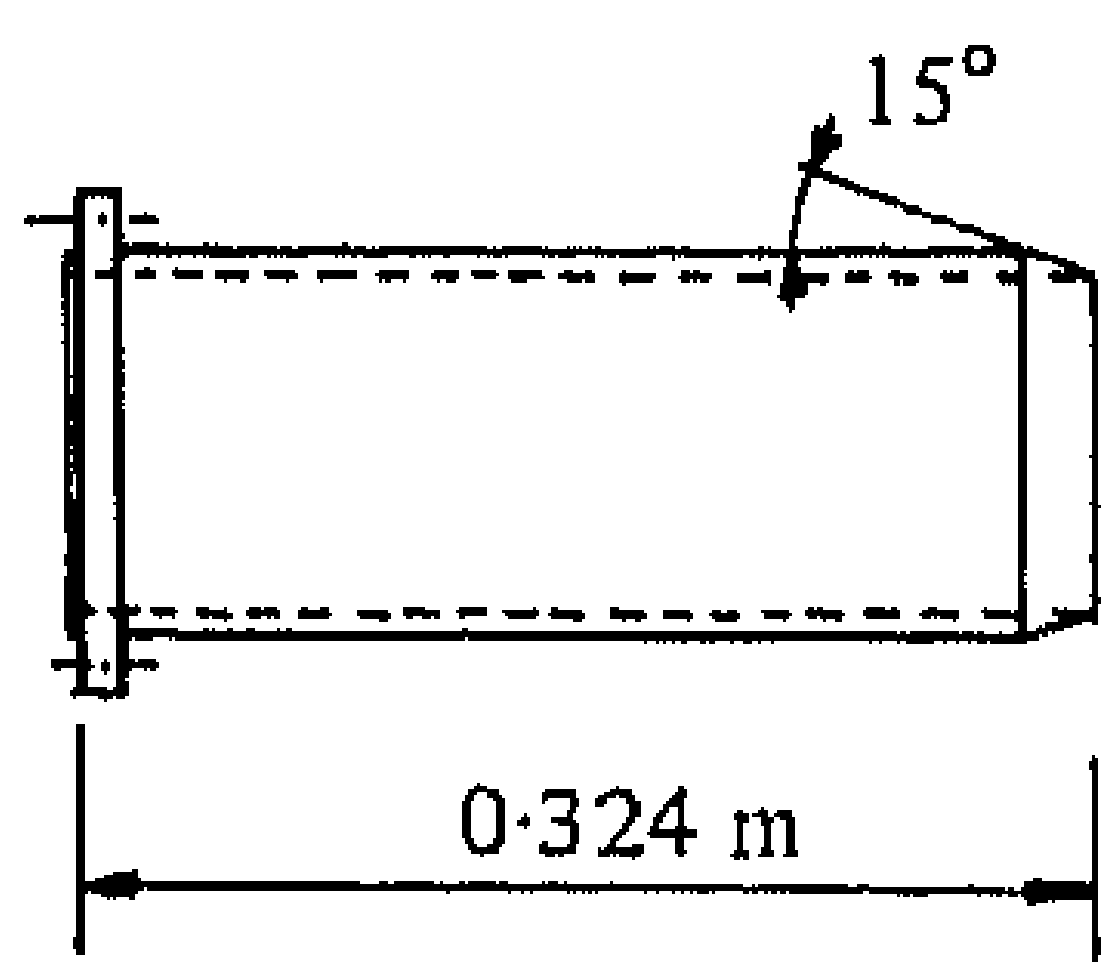

$(c)$

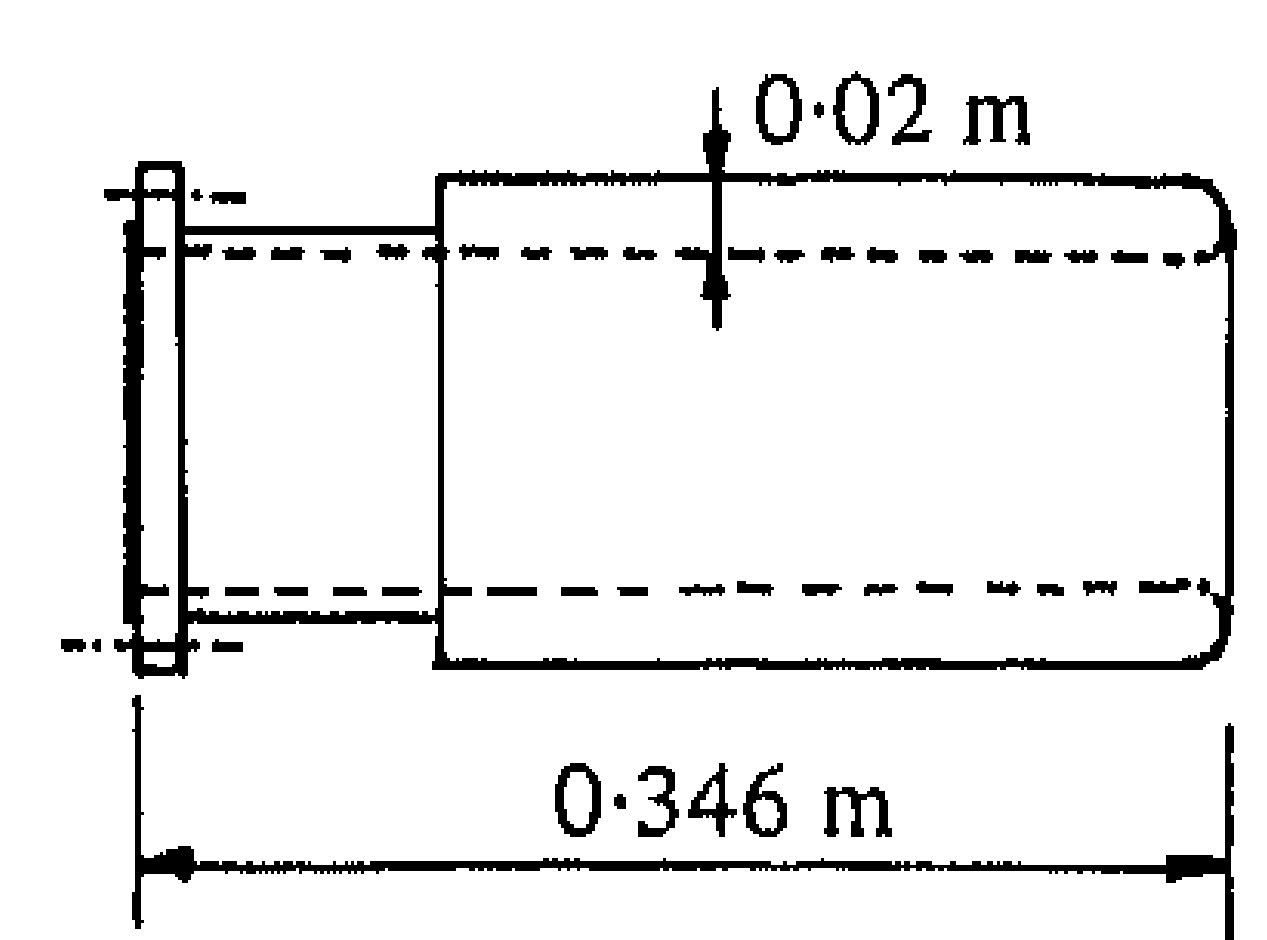

FIGURe 4. The dimensions of the sections of the open tube: (a) steel section; . (b) open end with sharp edge; (c) open end with round edge.

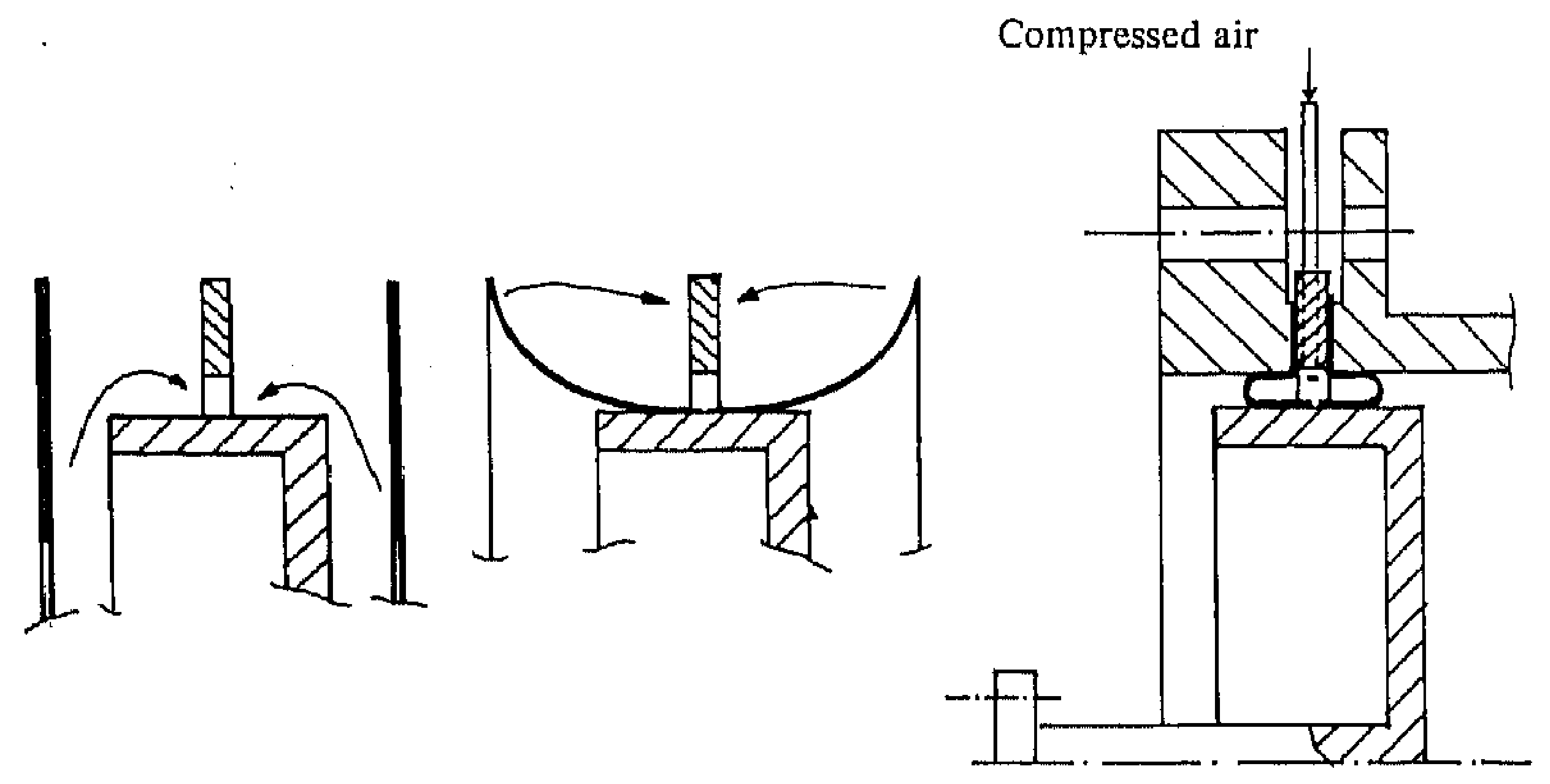

Figure 5. Construction of the piston sealing.

result (2.19) can be observed, confirming the experimental results in Van Wijngaarden (1968) which were for relatively large $\delta / R$.

Accordingly, the aim of this paper is primarily to investigate in more detail the behaviour of the flow near the exit in sharp-edged pipes for low values of $\delta / R$ and correspondingly large Strouhal number.

\section{Experimental set-up}

In this section we describe how the experiments were carried out. We used a tube consisting of steel sections of $1.5 \mathrm{~m}$ length with an internal diameter of $0.11 \mathrm{~m}$, as shown in figure 4 . The end sections, both with sharp and with round edges, were made of Perspex. The radius of curvature of the smooth edge is $0.01 \mathrm{~m}$. The oscillations in the pipes were excited by a piston at the other end. The problem of making a good sealing between the piston and the pipe wall was solved as shown in figure 5 . The fairly large clearance $\dagger$ is filled up with a kind of tyre which can be pressurized to an absolute pressure of about 1.5 bar. This tyre, rubber with a thickness of $0.007 \mathrm{~m}$, is glued to the piston, as indicated in figure 5 , and clamped to the end of the pipe. The leakage through this sealing is negligible. Two other advantages of this construction are that there is only a small friction force between tube and piston and that the construction poses no severe lining problems with the excitation, an MB electro-

$\dagger$ Radial modes generated at the piston as a result of this clearance are strongly attenuated because the cut-off frequency, of order $a_{0} / R$, is much larger than the resonance frequency, given in (2.2), 
dynamic shaker. With this device we could carry out experiments with piston amplitudes $\delta$ as small as $10^{-5} \mathrm{~m}$ without distortion by friction. The amplitude of the piston was measured both with a Peekel inductive displacement inducer and with an accelerometer built in the excitator.

The pressure $p_{p}$ at the piston was measured with a Bruel \& Kjaer $\frac{1}{8}$ microphone located at a distance of about $0.03 \mathrm{~m}$ from the piston. This distance is very small with respect to the wavelength: the latter is four times the pipe length, which varies, see figure 3 , between 1 and 6 metres. Moreover there is an antinode of the pressure at the piston under resonance. The pressure at $0.03 \mathrm{~m}$ from the piston therefore differs negligibly from the pressure at the piston. The pressure at the exit of the pipe was measured with the same type of microphone. Here a problem arises. In the most simple kind of linear theory the pressure is zero at the open end. This however is not true, because due to radiation from the open end (Levine \& Schwinger 1948) the firstorder standing wave has a node not at the exit but at a distance 0.61 times the radius outside the pipe. So, when one measures the pressure right at the exit, this signal contains, apart from second-order quantities as in (2.14) and (2.16), a first-order contribution. Bearing this in mind we acted as follows: with a microphone the pressure was measured at various locations inside the tube, indicated with distance $x$ from the mouth. These measured results were Fourier analysed and the Fourier components were determined as a function of $x$. With help of these the pressure was extrapolated to a value at $x=0.61 R$. In the calculation of the Fourier components the measurements made near the exit were weighted less heavily than those further inside the tube because of the inhomogeneity of the pressure across the pipe near the exit. The velocity can be obtained from the pressure by the relation

$$
\rho_{0} \frac{\partial u}{\partial t}+\frac{\partial p}{\partial x}=0
$$

between pressure and velocity, which is valid up to the third order in $u$ because the first-order $\partial u / \partial x$ is zero at the mouth.

Experimental analysis of flow behaviour near the edge. In order to make the flow near the edge at the exit visible we connected to the circular tube a two-dimensional open end with the same area of cross-section. Visualization was accomplished with schlieren methods, the necessary density gradients being realized by slightly heating the upper of the two plates forming the two-dimensional channel. This set-up is shown in figure 6 . Because of the heating the streak line originating from the edge can be made visible. For other streak lines thin hot wires may be used. Two versions of the schlieren method were used, illustrated in figures 7 and 8. In both of these, each point $I$ in the object plane is illuminated by a narrow beam coming from the light source $S$. The camera is focused on this object plane. This means that all rays from $I$ come together at one point of the film $F$, even if the beam is refracted, as in $I_{1}$, by some disturbance. In the first version, the conventional schlieren method, a knife edge $E$ is placed in the image of the aperture $A$, figure 7 . In the second version there is instead a Wollaston prism $W$ and two polarization filters $G 1$ and $G 2$. The Wollaston prism splits a ray into two slightly diverging components. This means that the light falling on a point $I^{\prime}$, see figure 8 , stems from two different points $I_{1}$ and $I_{2}$ in the object plane. One is polarized in the plane of the paper of figure 8 , the other perpendicular to this. The filter $G 1$ polarizes the light over an angle $\frac{1}{4} \pi$ with respect to the plane of the 


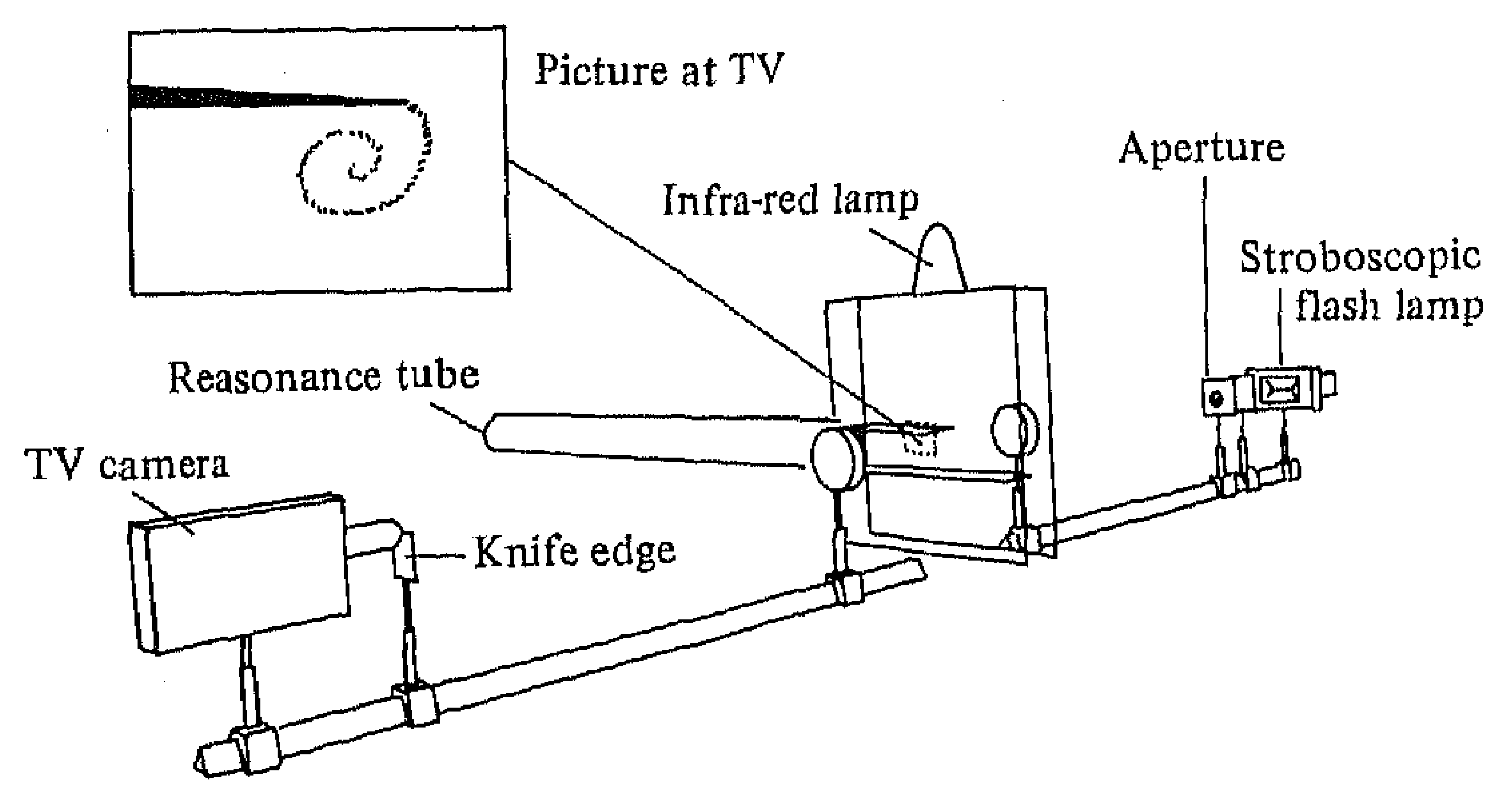

Fiaure 6. Experimental set-up for the flow visualization.

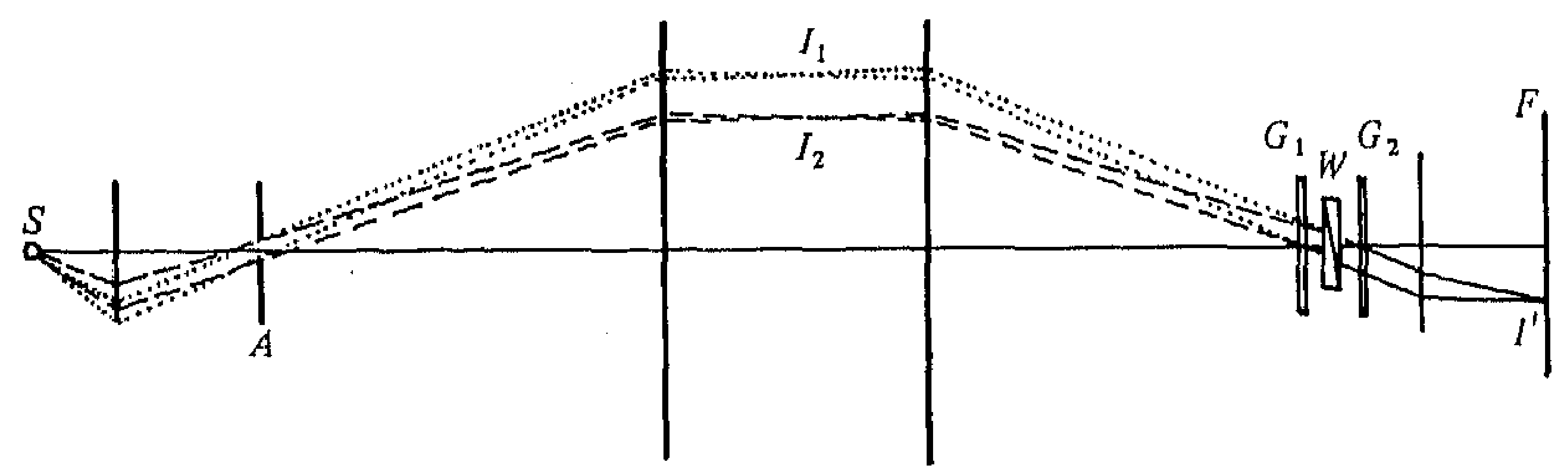

Fraurm 7. Sohematic diagram for conventional schlieren method.

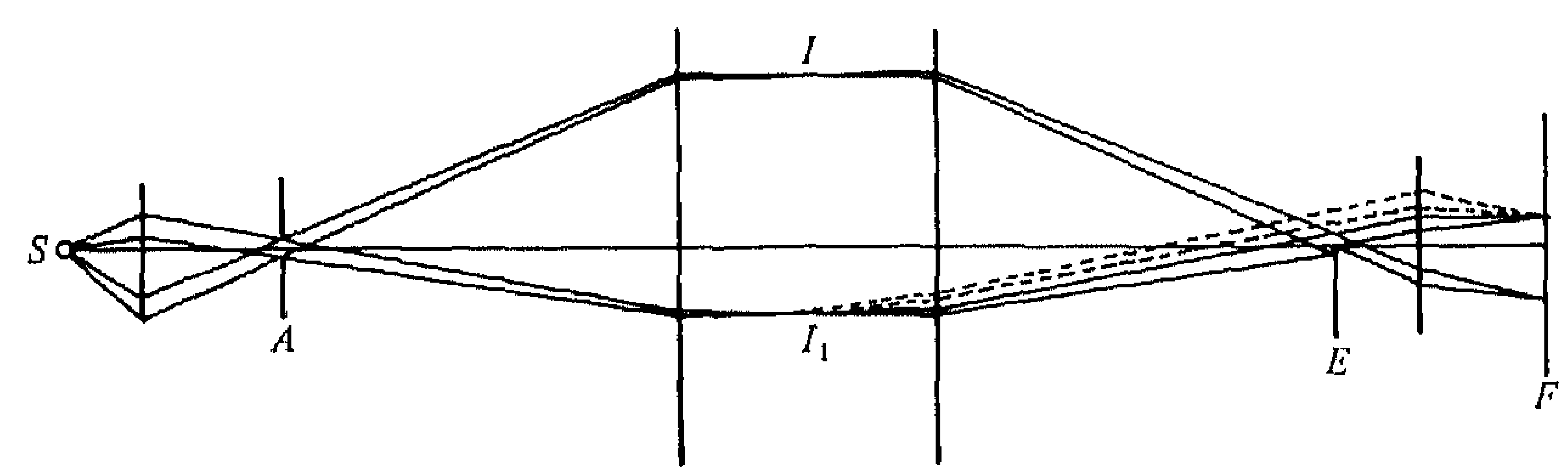

FIGURm 8. Schematic diagram for schlieren method with Wollaston prism.

paper and renders the two components behind the prism of equal strength. The filter $G 2$ polarizes the two components in the same direction (perpendicular to $G 1$ ), making interference possible. This interference depends on the relative phase shift of the two rays. In the absence of flow inhomogeneities this phase shift is determined by the location at which the rays pass the Wollaston prism. If the prism has a neutral position the interference is destructive for all wavelengths. By slight shifting of prism some background colour can be established, as shown in figure 13, which has been obtained in this way. An additional phase shift is established when the two rays pass through different densities in $I_{1}$ and $I_{2}$. This additional phase shift results in a change of colour as shown in figure 13 . This plate together with figure 12 obtained with the conventional schlieren method will be discussed in the next section, which deals with experimental results. 


\section{Experimental results}

Experimental results obtained at resonance for the pressure at the piston were shown already in figure 3 , in terms of the quantity $Q$ defined by $(2.10)$. For the determination of the pressure near the exit, the pressures measured at various locations $x$ within the pipe were (in the way described in $\S 3$ ) extrapolated to the cross-section $x=0.61 R$ outside the pipe; this because the first-order standing wave has a pressure node there so that second-order quantities like $\rho_{0} u^{2}$ are not drowned in the first-order signal. Results of this are shown in figures $9-11$. In figures $9(a)-(f)$, for $S t=0.73$ and $K=0.026$, the pressure is plotted as a function of time during one cycle at various locations $x$ inside the tube. The pressure obtained by extrapolation for $x=0.61 R$ is represented, together with the velocity, in figure $9(g)$. There is clearly a significant difference between the pressure right at the exit, $x=0$, and at $x=0.61 R$. Also there is, in the pressure at $x=0.61 R$, apart from a contribution from a third harmonic (for which we bave no explanation), a tendency to variation like $u^{2}$, as occurring in the boundary condition (2.16). A good agreement with (2.16) cannot be expected because the Strouhal number is not really small. Another set of measurements of this type is given in figures $10(a)-(c)$, this for $K=0.026$ and $S t=0.29$. In figures $10(a, b)$ the measured profiles are shown, in figure $10(c)$ the extrapolation to $x=0.61 R$ and the measured velocity. The same features as in the preceding figure can be observed.

This kind of experiment was also carried out with pipes provided with a smooth edge. A result for $K=0.026$ and $S t=0.41$ is shown in figures $11(a)-(c)$. The 'Bernoulli term', $-\frac{1}{2} \rho_{0} u^{2}$, in (2.14) is clearly visible, although here also the Strouhal number is too large for the flow to be quasi-steady. The main conclusion to be drawn from these measurements is that, if the boundary condition at the open end is applied at $x=0.61 R$, then, in that boundary condition, only effects other than acoustic radiation need to be taken into account.

Both qualitative and quantitative information about vortex formation near the edge, already observed by Van Wijngaarden \& Wormgoor (1974), was obtained from the schlieren pictures. In figure 12 (plate 1 ) is shown the rolling-up, during one cycle of the flow, of the vortex sheet leaving the edge. These pictures have been made in the two-dimensional end section described in $\S 3$. In each picture the time at which it was taken is indicated as fraction of the period $T$ of the motion. The velocity, measured with hot wires, is given with the help of arrows, the length of which is a measure for the magnitude of the velocity; the direction is as indicated by the arrows. The process of formation of the rolled-up vortex and the subsequent expulsion of this vorticity from the pipe can be followed in detail. The Strouhal number, based on the distance between the plates, is large, $S t=8$.

Apart from the vortex formation several other interesting features of the flow are visible. Notice first the inhomogeneity of the velocity distribution at the exit. At $t=0.45 T$ the flow near the edge is already directed outward, while nearer the centreline air is still flowing in. On the other hand, at $t=0.96 T$ air is leaving near the centre but already flowing in at the edge. Further, it is of interest to know whether or not a Kutta condition is satisfied by the flow at the edge. Detailed study of enlarged versions of the pictures shown in figure 12 have led us to the conclusion that streamlines leave the edge smoothly in the sense that, at each instant, the streamline leaving the edge coincides with the tangent to the edge. 


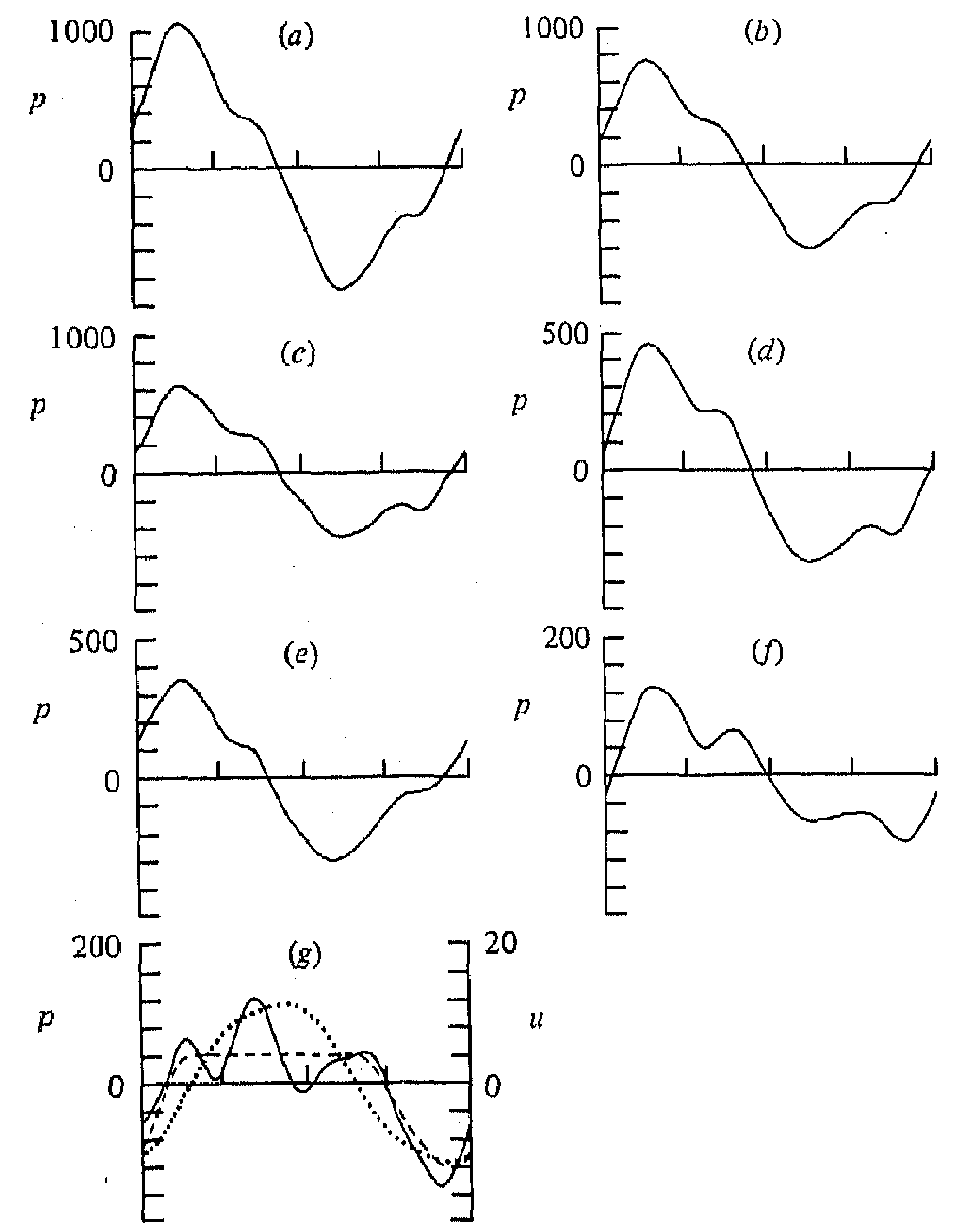

Figure 9. The pressure near the open end of the tube, $p-\bar{p}, \bar{p}$ being the average value over a period, as a function of time for the sharp edge. Measurements: $(a) x=-6 R ;(b) x=-4 R$; (c) $x=-3 R ;(d) x=-2 R ;(e) x=-R ;(f) x=0 .(g) x=0.61 R:$ - extrapolated pressure; $\ldots .$. , velocity ;,-- , pressure calculated with (2.16). The pressure $p-\bar{p}$ is in $\mathrm{N} \mathrm{m}^{-2}$, the velocity in $\mathrm{ms}^{-1}$.

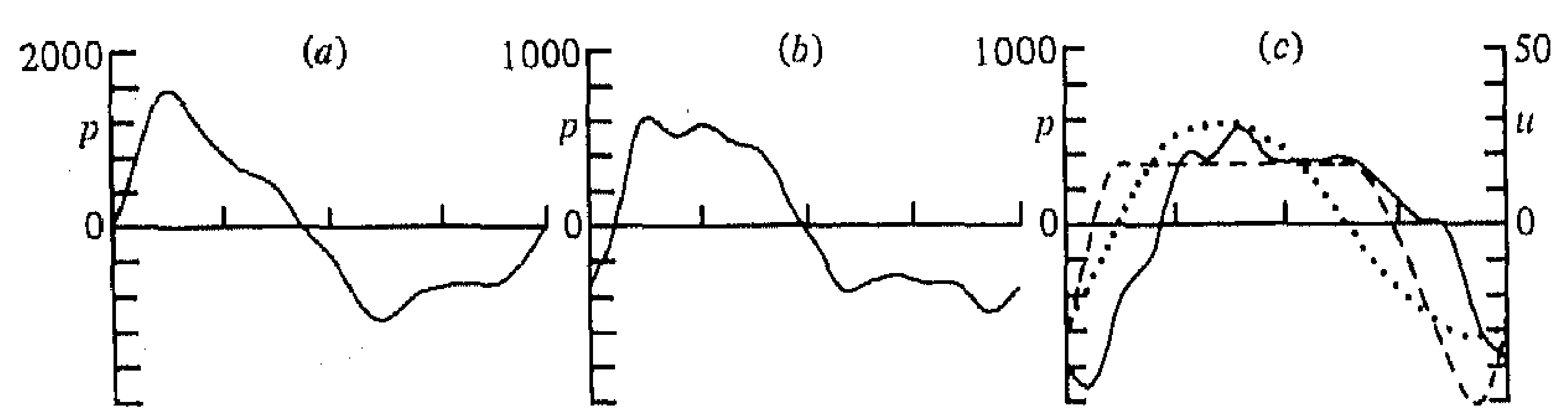

Fraure 10. The pressure near the open end of the tube, $p-\bar{p}, \bar{p}$ being the average value over a period, as a function of time (sharp edge). Measurements: (a) $x=-3 \cdot 6 R ;(b) x=-1 \cdot 6 R$. (c) $x=0.6 R:-$, extrapolated pressure; $\cdots . .$. , velocity; - . , pressure calculated from (2.16). The pressure $p-\bar{p}$ is in $\mathrm{N} \mathrm{m}^{-2}$, the velocity in $\mathrm{ms}^{-1}$.

The rolling-np process of the vortex sheet leaving the edge, is also very clearly visible in the pictures taken with help of the Wollaston prism. Figure 13 (plate 2) shows a series of such pictures. From these and those shown in figure 12 it follows that a vortex sheet leaves the edge and subsequently rolls up during inflow quite near the edge. The displacement of the centre of the vorticity-containing region is small during 


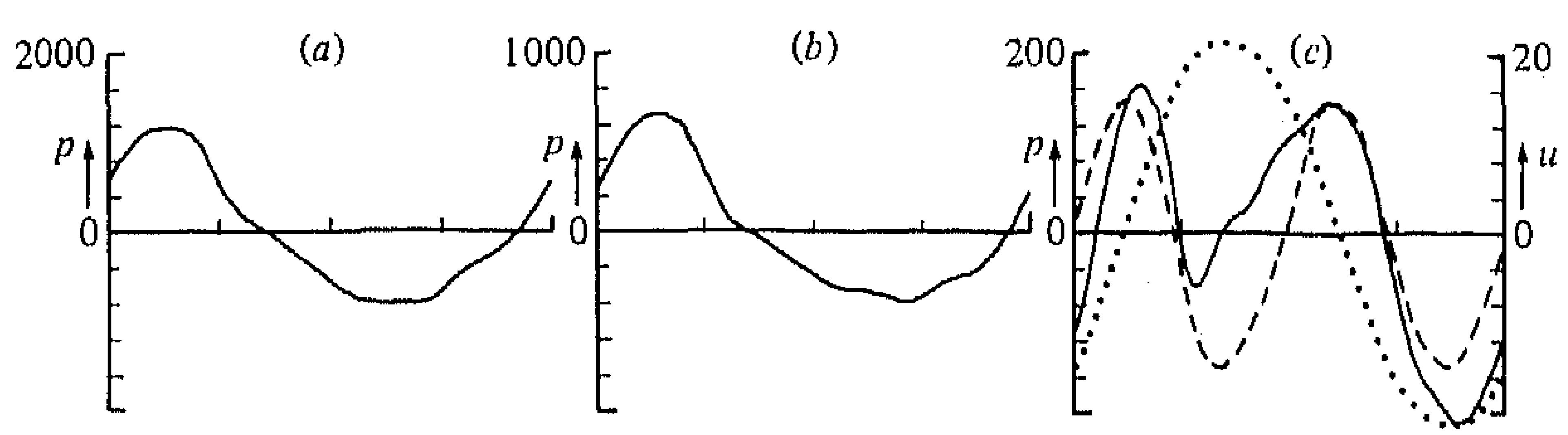

Frguize 11. The pressure near the end of the tube, $p-\bar{p}, \bar{p}$ being the average value over a period, for the round edge as a function of time. Measurements: $(a) x=-3 \cdot 4 R ;(b) x=-1 \cdot 4 R$. (c) $x=0.6 R:-$, extrapolated pressure; $\cdots \cdots$, velocity; $-\ldots$, pressure calculated from (2.14) applied both at inflow and at outflow. The pressure $p-\bar{p}$ is in $\mathrm{N} \mathrm{m}^{-2}$, the velocity in $\mathrm{m} \mathrm{s}^{-1}$.

(a)

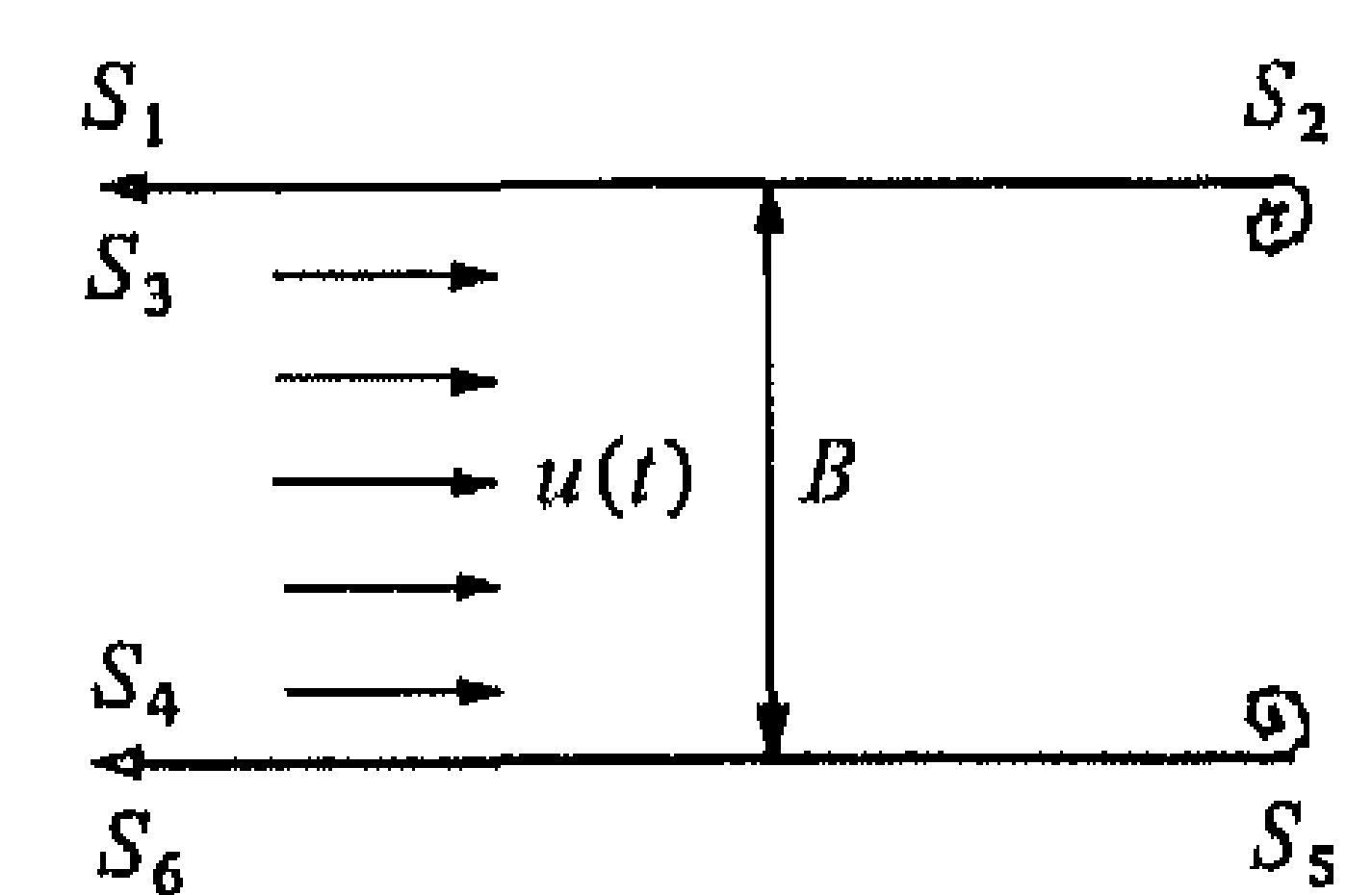

(b)

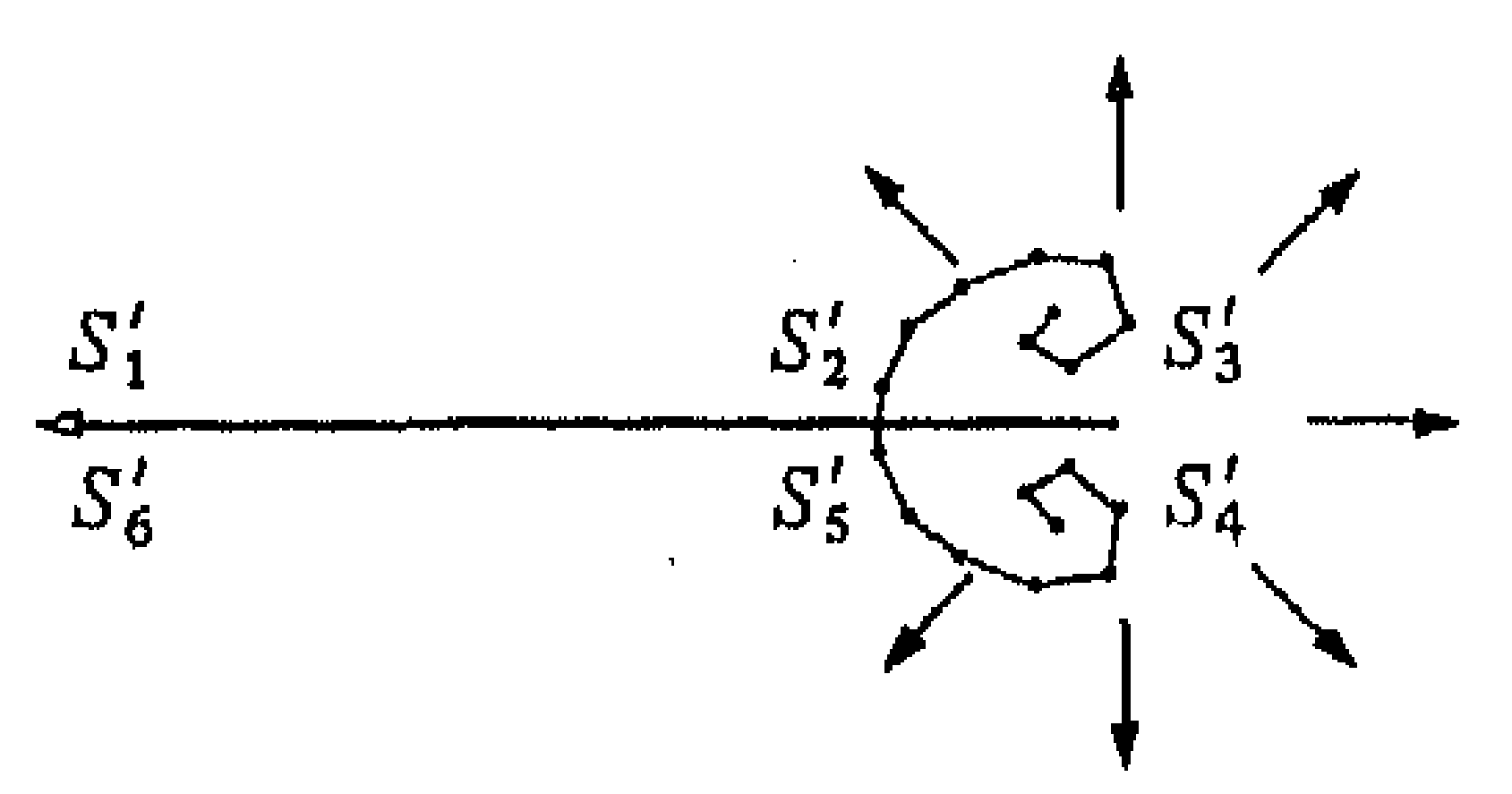

FraUre 14. The conformal transformation of a semi-infinite channel with two rolled-up vortices. (a) The $z$ plane. (b) The $\zeta$ plane.

inflow but becomes large during outflow. It can be anticipated that this process will have an important feedback on the amplitude of the standing wave in the tube. That is why we tried to develop a mathematical model of the vortex motion near the edge, on the basis of observations of which figures 12 and 13 are typical examples.

\section{Mathematical model for the flow near the edge}

We consider the two-dimensional flow near the edge as depicted in figure $14(a)$. The vorticity-containing region of the pipe has very small dimensions with respect to the wavelength and the flow in this region may be considered as incompressible. Viscosity is neglected, apart from its effect on boundary conditions (the Kutta condition). In the channel, as $z \rightarrow-\infty$, is a uniform time-dependent flow with velocity $u(t)$ which in our case will be specified later as $\hat{u} \sin t$. From the edges, $z=0$ and $z=i B$, vortex sheets develop and gradually roll up after the motion has been started. Once the location of the vortex sheets with their distribution of vorticity is known, the velocity and pressure at each point of the $z$ plane can be calculated. Eventually our aim is to calculate the pressure difference, $\Delta p_{v}$ say, between the wave in the tube as $z \rightarrow-\infty$ and the outside atmosphere, insofar as this difference is caused by the presence of the vortex sheets. To accomplish this we first map the $z$ plane cut along

$$
\left.\begin{array}{ll}
y=0 & (-\infty<x<0), \\
y=B & (-\infty<x<0),
\end{array}\right\}
$$

on a $\zeta$ plane cut along the real axis from $-\infty$ to 1 , by

$$
\frac{z}{B}=\frac{1}{2 \pi}\{\ln (1-\zeta)+\zeta\}
$$


The way in which this transformation maps the channel bounded by the lines given in $(5.1)$ on the $\zeta$ plane is indicated in figure $14(b)$. In particular the region deep down the channel, $z \rightarrow-\infty$, is mapped into $\zeta=1$ and hence the uniform flow in the $z$ plane becomes a source at $\zeta=1$ in the $\zeta$ plane. The vortex sheets coming from the edges at $z=0$ and $z=i B$ are mapped as vortex sheets coming from the origin in the $\zeta$ plane, in complex-conjugate positions.

For the subsequent (numerical) calculation each vortex sheet is divided into a finite number of discrete vortices. Let $w$ be the complex potential due to $N$ vortices and their images and the source at $\zeta=1$. The trajectory of any individual vortex with circulation $\Gamma_{n}$ is given by

$$
\frac{\overline{d z_{n}}}{d t}=\lim _{z \rightarrow z_{n}}\left\{\frac{d w}{d z}-\frac{\Gamma_{n}}{2 \pi i} \frac{1}{z-z_{n}}\right\}
$$

the bar denoting a complex conjugate. In terms of $\zeta,(5.3)$ reads

$$
\frac{\overline{d z_{n}}}{d t}=\left(\frac{d \zeta}{d z}\right)_{\zeta \rightarrow \zeta_{n}} \lim _{\zeta \rightarrow \zeta_{n}}\left[\frac{d w}{d \zeta}-\frac{\Gamma_{n}}{2 \pi i}\left\{\frac{1}{\zeta-\zeta_{n}}-\frac{1}{2}\left(\frac{d^{2} z / d \zeta^{2}}{d z / d \zeta}\right)\right\}\right] .
$$

Given the initial position of the vortices their motion can be calculated with help of this relation. However, the total vorticity $\Gamma_{\text {tot }}$ is not a constant because vorticity is continuously generated at the edges. The rate at which vorticity is generated at each of the edges $z=0, z=i B$, is given by

$$
\frac{d \Gamma_{\text {tot }}}{d t}=\frac{1}{2}\left\{\left(\frac{\overline{d w}}{d z}\right)_{+}+\left(\frac{\overline{d w}}{d z}\right)_{-}\right\}\left\{\left(\frac{\overline{d w}}{d z}\right)_{+}-\left(\frac{\overline{d w}}{d z}\right)_{-}\right\} .
$$

In this relation the subscripts + and - refer to the sides of the vortex sheet leaving the edge. The resulting potential $w$ must satisfy the Kutta condition at the edges. Because the transformation (5.2) is singular at these points, this means in the $\zeta$ plane that

$$
\frac{d w}{d \zeta}=0 \quad \text { at } \quad \zeta=0
$$

Because the problem of determining $w$ and the geometry of the vortex sheets can not be solved analytically, recourse to numerical computation must be made for this and similar problems. For a survey of the various numerical methods used we refer to recent papers by Clements \& Maull (1976) and Saffman \& Baker (1979). Owing to the discretization of the vortex sheets, errors are introduced numerically which accumulate and eventually tend to destroy a coherent flow pattern. This tendency of the collection of discrete vortices to form together a chaotic flow pattern becomes stronger when the number of vortices is increased. However, limiting the number of line vortices, we succeeded in calculating the pressure difference $\Delta p_{v}$ as a function of time in the case of a harmonic motion $\hat{u} \sin t$ in the tube for $t>0$. Details of this calculation are given in the next section.

\section{Numerical calculation of the motion of the vortex sheet}

Each of the vortex sheets is represented by $N$ discrete vortices of circulation $\Gamma_{n}, n=1, \ldots, N$. In the $\zeta$ plane, the vortices belonging to the sheet coming from $z=i B$ are situated at $\zeta=\zeta_{n}$; those belonging to the sheet coming from $\zeta=0$ are at $\zeta=\bar{\zeta}_{n}$. 
$(a)$

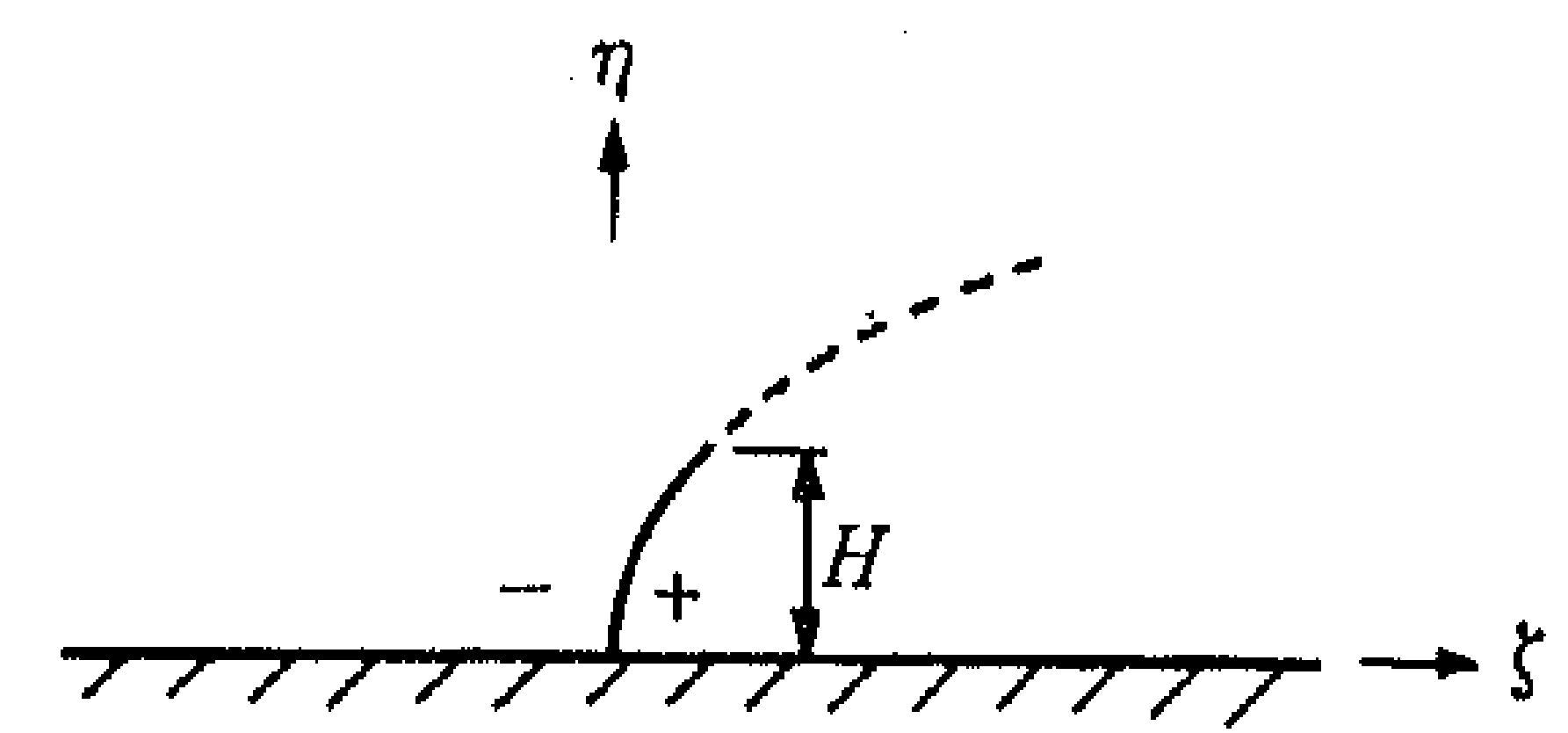

(b)

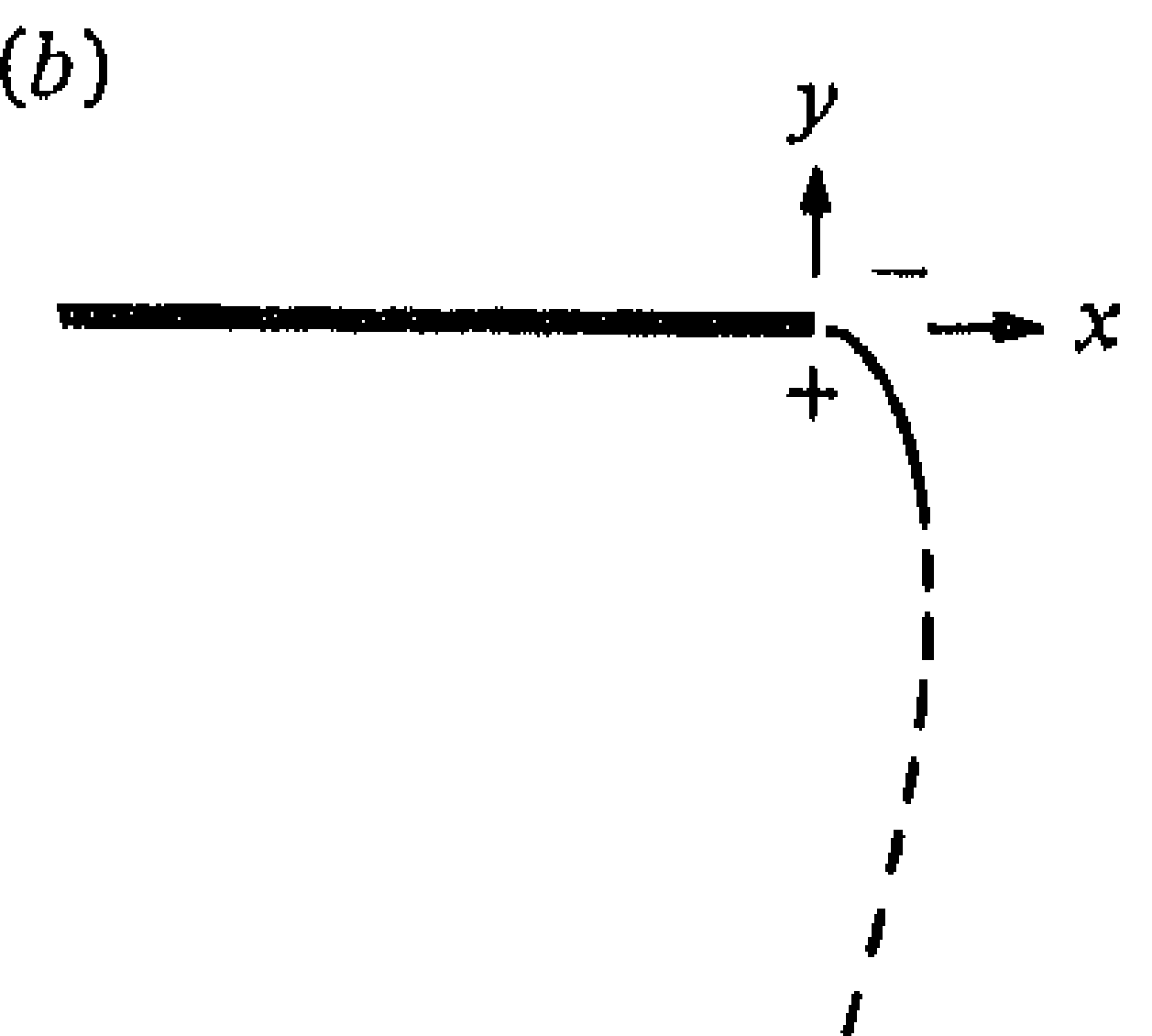

FigUre 15. The mapping of a vortex sheet with a parabolic shape in the $\zeta$ plane $(a) ;(b)$ the $z$ plane.

The first vortex, with circulation $\Gamma_{1}$, is distributed along a small vortex sheet, the shape of which is chosen in the $\zeta$ plane as

$$
\zeta=\xi+i \eta=\xi_{2} \eta^{2} / \eta_{2}^{2}+i \eta
$$

This parabolic shape has been chosen because, upon transformation to the $z$ plane, see figure 15 , it resembles quite closely the shape of the beginning of the vortex sheet observed in the experiments as shown for example in figure 13. This representation of the first vortex by a sheet element with a continuous vorticity distribution, $\gamma$ say, enables us to apply the relation (5.5) for vortex generation in a convenient way. In a first approximation the vorticity is constant along the sheet element in the physical plane. Since it follows from (5.2) that near $\zeta=0$ we have $d z / d \zeta=-\zeta B / 2 \pi+O\left(\zeta^{2}\right)$, the $\gamma$ distribution is linear in the $\zeta$ plane,

$$
\gamma=\frac{d \Gamma}{d \zeta}=\frac{d \Gamma}{d z} \frac{d z}{d \zeta}=c \zeta+O\left(\zeta^{2}\right)
$$

$c$ being a constant. Assuming the curvature of the sheet element to be small,

we can write

$$
\xi_{2} \ll \eta_{2},
$$

$$
d \Gamma=\frac{2 \Gamma_{1}}{H^{2}} \eta d \eta
$$

$H$ being the height (see figure 15) of the element.

In the $\zeta$ plane the resulting potential is due to a source of strength $u B$, when $u$ is the velocity far inside the pipe, the $N-1$ discrete vortices with strength $\Gamma_{n}$, their images with strength $-\Gamma_{n}$ and finally the small vortex sheets near $\zeta=0$. The resulting complex velocity is, with use of (5.2) and (6.4), found to be given by

$$
\frac{d w}{d z}=\frac{1-\zeta}{\zeta B}\left[\frac{u B}{1-\zeta}+i \sum_{n=2}^{N} \Gamma_{n}\left\{\frac{1}{\zeta-\zeta_{n}}-\frac{1}{\zeta-\bar{\zeta}_{n}}\right\}+\frac{2 i \Gamma_{1}}{H^{2}} \int_{-I I}^{I I} \frac{\eta d \eta}{\zeta-i \eta-\xi_{2} \eta^{2} / \eta_{2}^{2}}\right]
$$

The Kutta condition (5.6) requires that $d w / d z$ be finite at $\zeta=0$. Evaluating the quantity in square brackets for $\zeta \rightarrow 0$ gives, using the approximation (6.3),

$$
u B-i \sum_{n=2}^{N} \Gamma_{n}\left(\frac{1}{\zeta_{n}}-\frac{1}{\bar{\zeta}_{n}}\right)-\frac{4 \Gamma_{1}}{H}=0
$$


Using $(5.5),(6.3),(6.5)$ and $(6.6)$, the rate of vorticity shedding is found to be

$$
\frac{d \Gamma_{\text {tot }}}{d t}=-\frac{4 \pi \Gamma_{1}}{H^{2}}\left[\frac{u}{B}+\sum_{n=2}^{N} \frac{\Gamma_{n}}{B^{2}} \frac{i\left(\zeta_{n}^{2}-\bar{\zeta}_{n}^{2}\right)}{\zeta_{n}^{2} \bar{\zeta}_{n}^{2}}-\frac{2 \xi_{2}}{\eta_{2}^{2}}\left\{\frac{u}{B}+\sum_{n=2}^{N} \frac{\Gamma_{n}}{B^{2}} \frac{i\left(\zeta_{n}-\bar{\zeta}_{n}\right)}{\zeta_{n} \bar{\zeta}_{n}}\right\}\right] .
$$

If the values of $\Gamma_{n}$ and $\zeta_{n}(2<n \leqslant N)$ and $\Gamma_{1}$ and $H$ are given at time $t$, their values at $t+\Delta t$ are calculated in three steps as follows:

(i) Calculation of the displacement of each vortex during the interval $\Delta t$. For this we use (5.4) and obtain, with help of (5.2),

$$
\begin{aligned}
\bar{z}_{n}(t+\Delta t)= & \bar{z}_{n}(t)+\Delta t \frac{1-\zeta_{n}(t)}{\zeta_{n}(t)}\left[\frac{u(t)}{1-\zeta_{n}(t)}+i \sum_{\substack{l=1 \\
l \neq n}}^{N} \frac{\Gamma_{l}}{B}\left\{\frac{1}{\zeta_{n}(t)-\zeta_{l}(t)}-\frac{1}{\zeta_{n}(t)-\bar{\zeta}_{l}(t)}\right\}\right. \\
& \left.-\frac{1}{2} \frac{i \Gamma_{n}}{B}\left\{\frac{2}{\zeta_{n}(t)-\bar{\zeta}_{n}(t)}+\frac{1}{\zeta_{n}(t)\left(1-\zeta_{n}(t)\right)}\right\}\right] .
\end{aligned}
$$

In calculating the contribution from the small vortex sheet with circulation $\Gamma_{1}$ wo have taken its centre $\zeta_{1}$ at $\eta_{1}=2 / 3 H$.

(ii) Shift of the indices of the vortex. This was done as follows:

$$
\begin{aligned}
& \Gamma_{n, \text { new }}=\Gamma_{n-1, \text { prev }} \quad(n=2,3, \ldots), \\
& \zeta_{n, \text { new }}=\zeta_{n-1, \text { nrev }},
\end{aligned}
$$

where 'prev' is shorthand for previous.

(iii) Calculation of the new vortex sheet circulation $\Gamma_{1}$ and its height $H$. The vorticity in $\Gamma_{1}$ is generated at a rate $d \Gamma_{\text {tot }} / d t$, so

$$
\Gamma_{1}=\Delta t \frac{d \Gamma_{\text {tot }}}{d t}
$$

Using (6.7) gives for $H$ the relation

$$
H^{2}=-4 \pi \Delta t\left[\frac{u}{B}+\sum_{n=2}^{N} \frac{\Gamma_{n}}{B^{2}} \frac{i\left(\zeta_{n}^{2}-\bar{\zeta}_{n}^{2}\right)}{\zeta_{n}^{2} \bar{\zeta}_{n}^{2}}-\frac{2 \xi_{2}}{\eta_{2}^{2}}\left\{\frac{u}{B}+\sum_{n=2}^{N} \frac{\Gamma_{n}}{B^{2}} \frac{i\left(\zeta_{n}-\bar{\zeta}_{n}\right)}{\zeta_{n} \bar{\zeta}_{n}}\right\}\right]
$$

From the Kutta condition (6.6) we finally obtain $\Gamma_{1}$.

In order to avoid an excessive growth of computation time as the calculation proceeds, an amalgamation process was introduced. The physical observation inspiring this is (see figure 13) the formation during each cycle of two spiral structures one with positive and one with negative vorticity. These spirals keep their identity, more or less, and may be represented by a single line vortex each. We chose the vortex with index $N_{0}$ as amalgamation centre. This means that as a rule its index was not shifted but that $\Gamma_{N_{0}-1}$ was absorbed by it. This continues until $\Gamma_{N_{0}-1}$ changes sign, in which case all indices are shifted. In absorbing $\Gamma_{N_{0}-1}$ the centre $\zeta_{N_{n}}$ moves to a new location $\zeta_{N_{0}, \text { new }}$, determined in our calculation in such a way that the potential far away inside the tube is least affected. The region far inside the tube is mapped in the $\zeta$ plane (see figure 14) near $\zeta=1$, where the potential due to a vortex with circulation $\Gamma_{a}$ in $\zeta_{a}$ is, together with its image in $\bar{\zeta}_{a}$, given by

$$
w \sim \frac{\Gamma_{a}}{2 \pi i} \ln \frac{1-\zeta_{a}}{1-\bar{\zeta}_{a}}+(1-\zeta) \frac{\Gamma_{a}}{2 \pi i}\left(\frac{1}{1-\zeta_{a}}-\frac{1}{1-\bar{\zeta}_{a}}\right) .
$$


Using this relation, the amalgamation was done such that the total circulation remains constant,

$$
\Gamma_{N_{0}, \text { new }}=\Gamma_{N_{0}, \text { prev }}+\Gamma_{N_{0}-1, \text { prev }}
$$

and that the right-hand side terms in (6.16) remain unchanged,

$$
\begin{aligned}
& \Gamma_{N_{0}, \text { new }} \arg \left(1-\zeta_{N_{0}, \text { new }}\right)=\Gamma_{N_{0}, \text { prev }} \arg \left(1-\zeta_{N_{0}, \text { prev }}\right)+\Gamma_{N_{0}-1, \text { prev }} \arg \left(1-\zeta_{N_{0}-1, \text { prev }}\right) \\
& \Gamma_{N_{0} \text {, new }}\left\{\frac{1}{1-\zeta_{N_{0}, \text { new }}}-\frac{1}{1-\bar{\zeta}_{N_{0}, \text { new }}}\right\}= \Gamma_{N_{0}, \text { prev }}\left\{\frac{1}{1-\zeta_{N_{0}, \text { prev }}}-\frac{1}{1-\bar{\zeta}_{N_{0}, \text { prev }}}\right\} \\
&+\Gamma_{N_{0}-1, \text { prev }}\left\{\frac{1}{1-\zeta_{N_{0}-1, \text { prev }}}-\frac{1}{1-\bar{\zeta}_{N_{0}-1, \text { prev }}}\right\} . \quad(6.14)
\end{aligned}
$$

The potential $w$ in the $z$ plane and its derivative $d w / d z$ given by (6.5) may with the help of Bernoulli's theorem be used to calculate the pressure far inside the tube, $z \rightarrow-\infty, \zeta \rightarrow 1$, and far outside the tube, $z \rightarrow \infty, \zeta \rightarrow \infty$. Taking the difference between these pressures and subtracting the pressure difference that would occur if no vortices were present, we obtain for the pressure difference due to the presence of the vortex sheets

$$
\Delta p_{v}=\frac{\rho}{\pi} \sum_{n=1}^{N} \frac{d}{d t}\left[\Gamma_{n}\left\{\arg \left(1-\bar{\zeta}_{n}\right)\right\}\right]
$$

The equations $(5.2),(6.6),(6.8),(6.9),(6.11)-(6.14)$ form a set with which a numerical simulation of the vortex shedding from the edges can be performed. We restrict ourselves here to low amplitudes of $u$. Then the displacement of a vortex during a cycle is relatively small and the vortex remains in the vicinity of the edge. This permits the approximation

$$
|\zeta| \ll 1 .
$$

Introducing this approximation enables to scale our variables in such a way that the scaled variables $\tilde{t}, \tilde{\zeta}, \tilde{z}, \tilde{H}, \tilde{u}, \widetilde{\Gamma}$ and $\Delta \tilde{p}_{v}$ are of unit order. The appropriate scaling is

$$
\left.\begin{array}{l}
\tilde{t}=\Omega t, \quad \tilde{\zeta}=\left(\frac{\Omega B}{\hat{u}}\right)^{\frac{1}{3}} \zeta, \quad \tilde{z}=(\Omega B / \hat{\imath})^{\frac{2}{3}}(z / B), \quad \tilde{H}=(\Omega B / \hat{\imath})^{\frac{1}{3}} H, \\
\tilde{u}=u / \hat{u}, \quad \Delta \tilde{p}_{v}=(\Omega B / \hat{u})^{\frac{5}{3}}\left(\Delta p_{v} / \rho \Omega^{2} B^{2}\right), \quad \tilde{\Gamma}=(\Omega B / \hat{\imath})^{\frac{4}{5}} \Gamma / \Omega B^{2} .
\end{array}\right\}
$$

With (6.16) and (6.17) the equations become

$$
\begin{aligned}
& \tilde{z}=-\frac{1}{4 \pi} \tilde{\zeta}^{2} \\
& \tilde{H}^{2}=4 \pi \Delta \tilde{t}\left\{\sum_{n=2}^{N} i \widetilde{\Gamma}_{n} \frac{\bar{\zeta}_{n}^{2}-\tilde{\zeta}_{n}^{2}}{\tilde{\zeta}_{n}^{2} \tilde{\zeta}_{n}^{2}}+\frac{2 \tilde{\xi}_{2}}{\tilde{\eta}_{2}^{2}}\left(\tilde{u}-\sum_{n=2}^{N} i \widetilde{\Gamma}_{n} \frac{\bar{\zeta}_{n}-\tilde{\zeta}_{n}}{\tilde{\zeta}_{n} \tilde{\zeta}_{n}}\right)\right\} ; \\
& \frac{4 \widetilde{\Gamma}_{1}}{\tilde{H}}=\tilde{u}+\sum_{n=2}^{N} i \tilde{\Gamma}_{n} \frac{\tilde{\zeta}_{n}-\bar{\xi}_{n}}{\tilde{\zeta}_{n} \tilde{\zeta}_{n}} ; \\
& \overline{\tilde{z}}_{n}(\tilde{t}+\Delta \tilde{t})=\overline{\tilde{z}}_{n}(\tilde{t})+\frac{\Delta \tilde{t}}{\bar{\zeta}_{n}(\tilde{t})}\left[\tilde{u}(\tilde{t})+i \sum_{\substack{l=1 \\
l \neq \neq}}^{N} \widetilde{\Gamma}_{l}\left\{\frac{1}{\tilde{\zeta}_{n}(t)-\tilde{\zeta}_{1}(t)}-\frac{1}{\tilde{\zeta}_{n}(\tilde{t})-\tilde{\zeta}_{l}(\tilde{t})}\right\}\right. \\
& \left.-\frac{1}{2} i \tilde{\Gamma}_{n}\left\{\frac{2}{\tilde{\xi}_{n}(\tilde{t})-\tilde{\xi}_{n}(\tilde{t})}+\frac{1}{\tilde{\zeta}_{n}(\tilde{T})}\right\}\right], \quad n=1,2, \ldots, N ;
\end{aligned}
$$


(a)

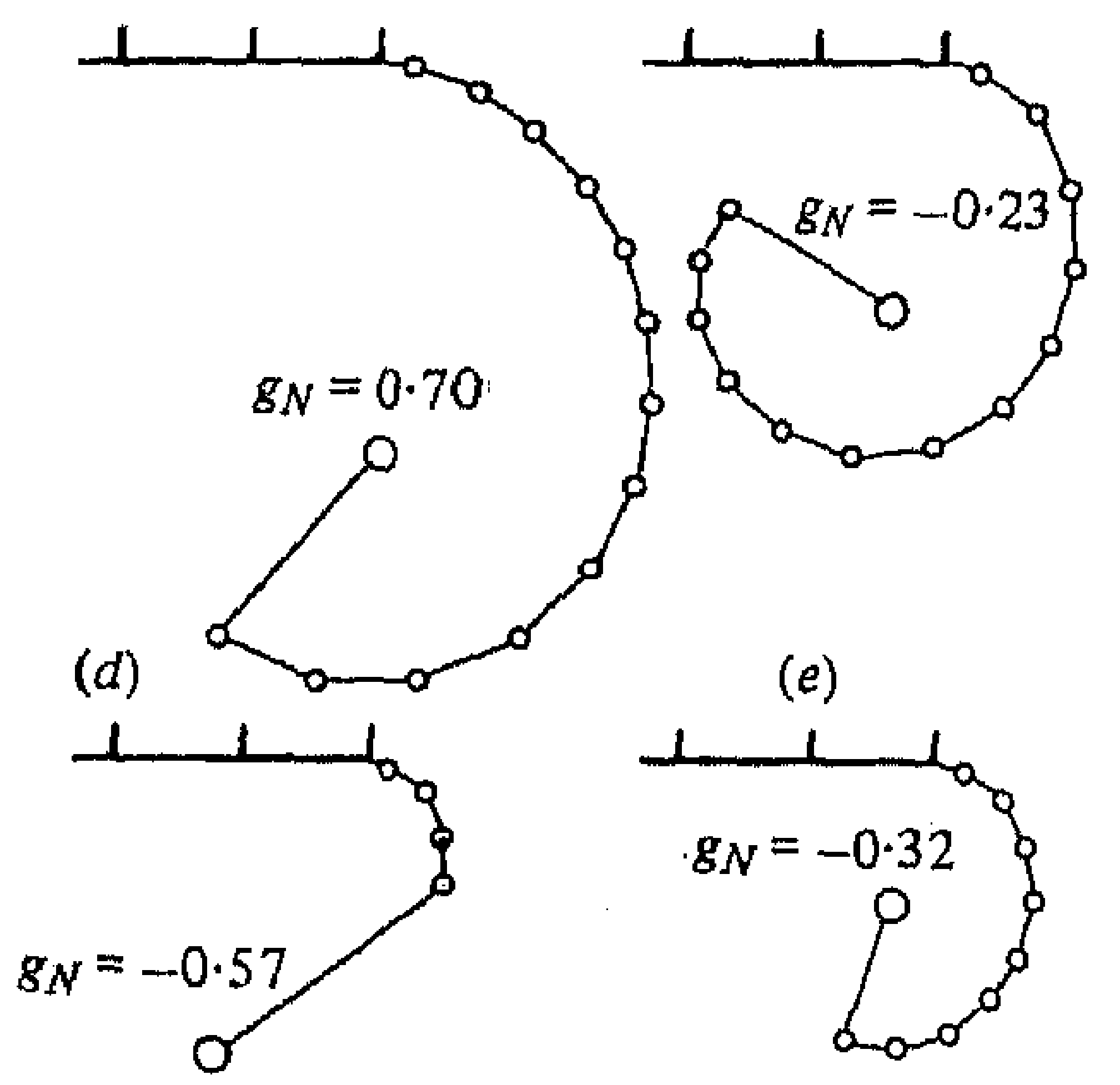

(c)
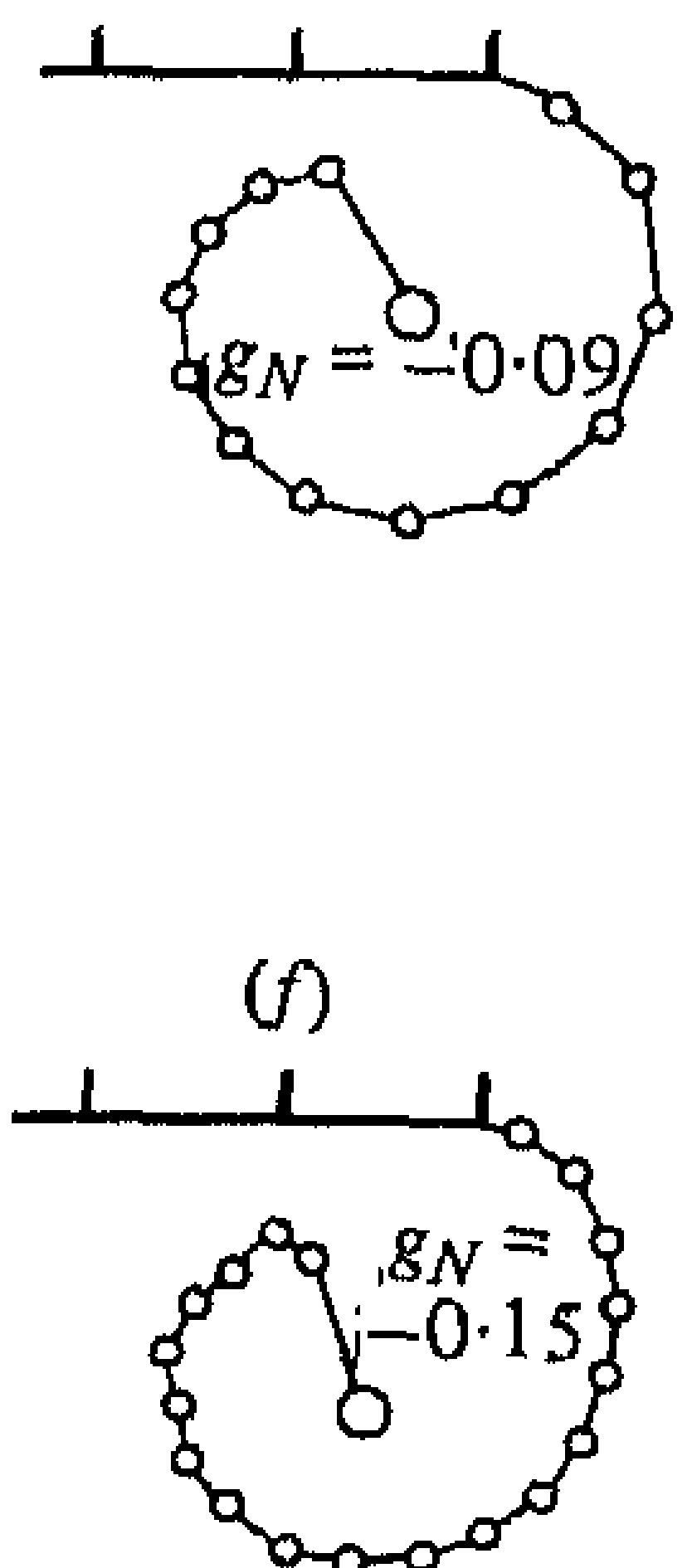

FIgURa 16. Some similarity solutions. (a) $\alpha=0, N=14, \Delta \tau=0.1 ;(b) \alpha=0.5, N=14$, $\Delta \tau=0.1 ;(a) \alpha=1, N=14, \Delta \tau=0.1 ;(d) \alpha=1, N=5, \Delta \tau=0.05 ;(e) \alpha=1, N=10$, $\Delta \tau=0.05 ;(f) \alpha=1, N=20, \Delta \tau=0.05$. The indicated length scale is 0.1 per division.

$$
\begin{aligned}
& \Delta \tilde{p}_{v}\left(\tilde{t}+\frac{1}{2} \Delta \tilde{t}\right)=\frac{1}{\pi \Delta t}\left[\sum_{n=1}^{N} \widetilde{\Gamma}_{n} \tilde{\eta}_{n}(\tilde{t}+\Delta \tilde{t})-\sum_{n=2}^{N} \widetilde{\Gamma}_{n} \tilde{\eta}_{n}(\tilde{t})\right] \\
& \widetilde{\Gamma}_{n, \text { new }}=\widetilde{\Gamma}_{n-1, \text { prev }} \quad \text { if } \operatorname{sgn} \widetilde{\Gamma}_{N_{0}}=\operatorname{sgn} \widetilde{\Gamma}_{N_{0}-1}, \quad n=2,3, \ldots, N_{0}-1 \text {; } \\
& \tilde{\zeta}_{n, \text { new }}=\xi_{n-1, \text { nrev }} \int \text { otherwise, } \quad n=2,3, \ldots, N+1 \text {; } \\
& N_{\text {new }}=N_{\text {prev }}+1 \text { if } \operatorname{sgn} \widetilde{\Gamma}_{N_{0}} \neq \operatorname{sgn} \widetilde{\Gamma}_{N_{0}-1} \text {; } \\
& \widetilde{\Gamma}_{N_{0}, \text { new }}=\widetilde{\Gamma}_{N_{0}-1, \text { prev }}+\widetilde{\Gamma}_{N_{0}, \text { prev }} \\
& \left.(\tilde{\Gamma} \tilde{\eta}\rangle_{N_{0}, \text { new }}=(\tilde{\Gamma} \tilde{\eta})_{N_{0}-1, \operatorname{prev}}+(\tilde{\Gamma} \tilde{\eta})_{N_{0}, \text { prev }}\right\} \quad \text { if } \operatorname{sgn} \widetilde{\Gamma}_{N_{0}}=\operatorname{sgn} \widetilde{\Gamma}_{N_{0}-1} \text {. } \\
& \left.(\tilde{\Gamma} \tilde{\xi})_{N_{0} \text { new }}=(\tilde{\Gamma} \tilde{\xi})_{N_{0}-1, \text { prev }}+(\tilde{\Gamma} \tilde{\xi})_{N_{0}, \text { prev }}\right)
\end{aligned}
$$

We applied the system of equations (6.22)-(6.28) first to compute a similarity solution. To obtain such a solution we write

$$
\tilde{u}=\tilde{t}^{\alpha}, \quad \tilde{\zeta}_{n}=\tilde{t}^{\beta_{1}}, \quad \tilde{z}_{n}=h_{n} \tilde{t}^{2 \beta_{1}}, \quad \Delta \tilde{t}=\tilde{t} \Delta \tau, \quad \tilde{\Gamma}_{n}=g_{n} t^{\rho_{2}}, \quad \Delta \tilde{p}_{v}=M t^{\beta_{3}},
$$

where $\alpha, \beta_{1}, \beta_{2}$ and $\beta_{3}$ are constants. Substituting the relations (6.25) gives for $\beta_{1}, \beta_{2}$ and $\beta_{3}$

$$
\beta_{1}=\frac{1}{3}(1+\alpha), \quad \beta_{2}=\frac{1}{3}(1+4 \alpha), \quad \beta_{3}=\frac{1}{3}(5 \alpha-1) .
$$

This result is in agreement with the similarity solution for the vortex sheet coming from a flat plate given by Prandtl, see Prandtl (1961). Since eventually we want to compute the solution for $\tilde{u}=-\sin \tilde{t}$, we took as starting condition the similarity solution for $\alpha=1$. The equations (6.18)-(6.24) were integrated with the substitutions $(6.25)$ and (6.26) until steady values for $s_{n}, h_{n}$ and $M$ resulted.

\section{Results}

In figure 16 some results of the calculation of the similarity solution are shown. Unfortunately the motion became chaotic when $N$ or $\Delta \tau$ were too large and also when 
(a)

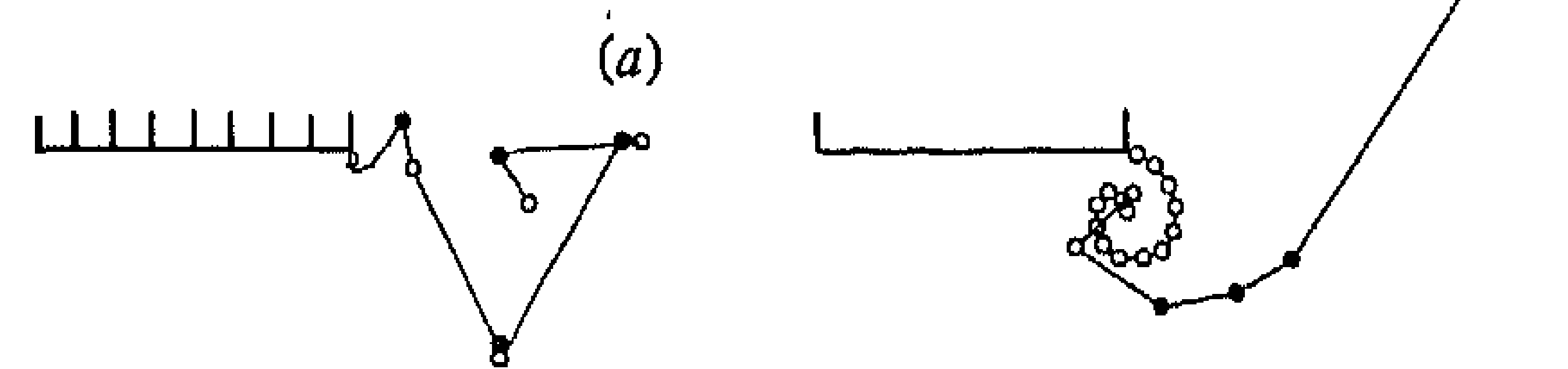

(b)
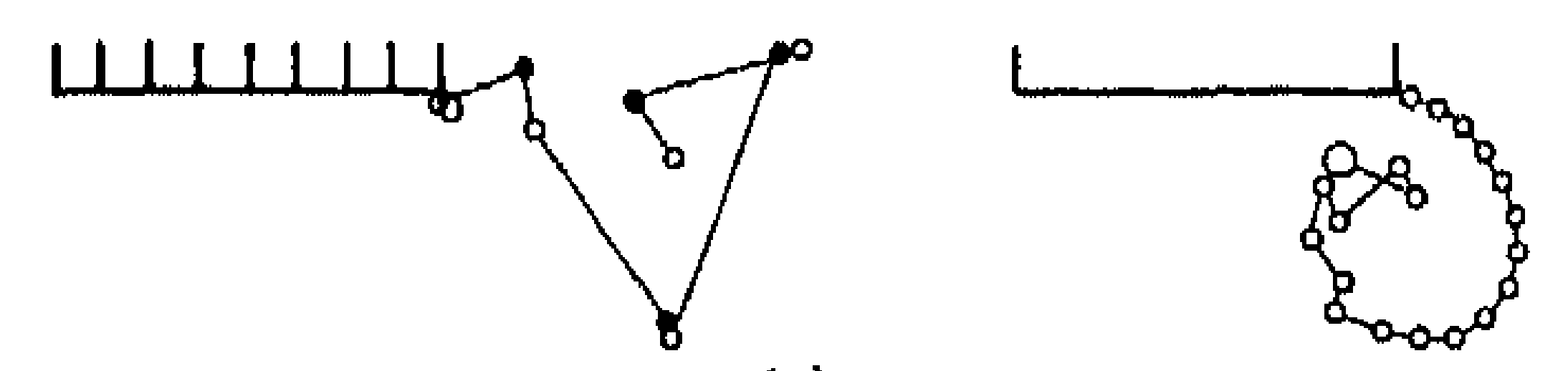

(c)
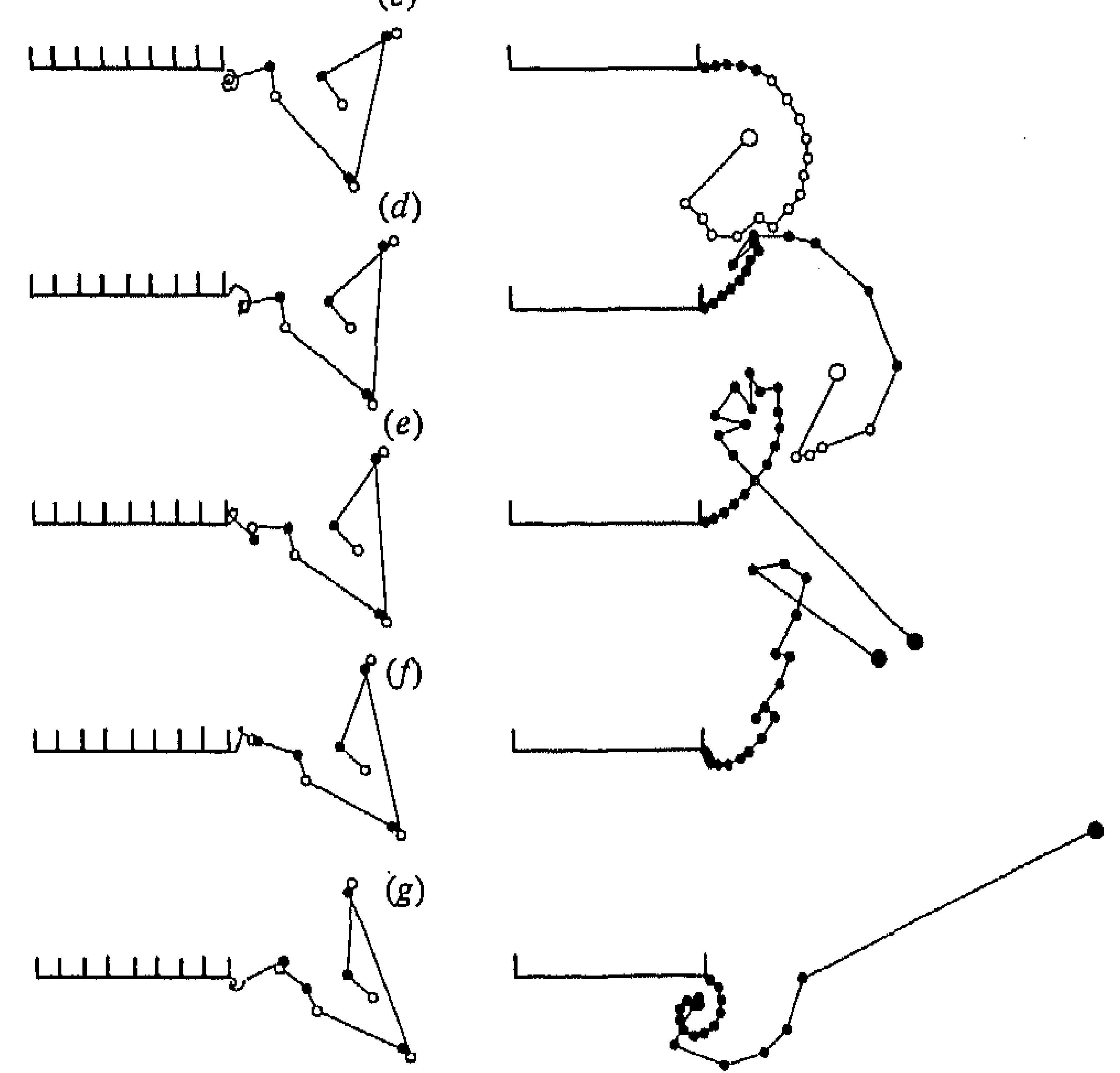

Fraure 17. The evolution of the vortex sheet for $N_{0}=20$. A.t the left the amalgamated vortex centres are shown. In the enlargement at the right the first $N_{0}$ vortices are shown. The indicated length scale is 1 per division. Open and closed circles indicate that the vortex strength is negative and positive respectively. (a) $\tilde{t}=26 ;(b) t=27 ;(c) t=28 ;(d) t=29 ;(e) t=30 ;(f) t=31$; (g) $t=32$.

$\Delta \tau$ was too small. We did not try to improve on this, because our interest in the similarity solution mainly is to use it as a start for the calculations with $\tilde{u}=-\sin t$. The results of these calculations are shown in figures 17 and 18. In the former the displacement of the vortices is shown during the fifth period after the start and $N_{0}=20$. At the left-hand side the positions of amalgamated vortices of negative strength (open circles) and positive strength (closed circles) are shown, whereas at the righthand side a more detailed picture is shown of the development near the edge of the plate. If we compare these results with the experimental observations in figures 12 and 13 , we see that there is qualitative agreement. The diameter of the vortex structure that is shed off is 0.9 whereas the scaled diameter in figure 12 is 0.73 , which is about $20 \%$ less.

In figure 18 the scaled pressure $\Delta \tilde{p}_{v}$, defined in (6.21), is given as a function of $\tilde{u}$, also defined in (6.21), for the case $\tilde{u}=-\sin \tau$. After one cycle the pressure $\Delta \tilde{p}_{v}$ was 


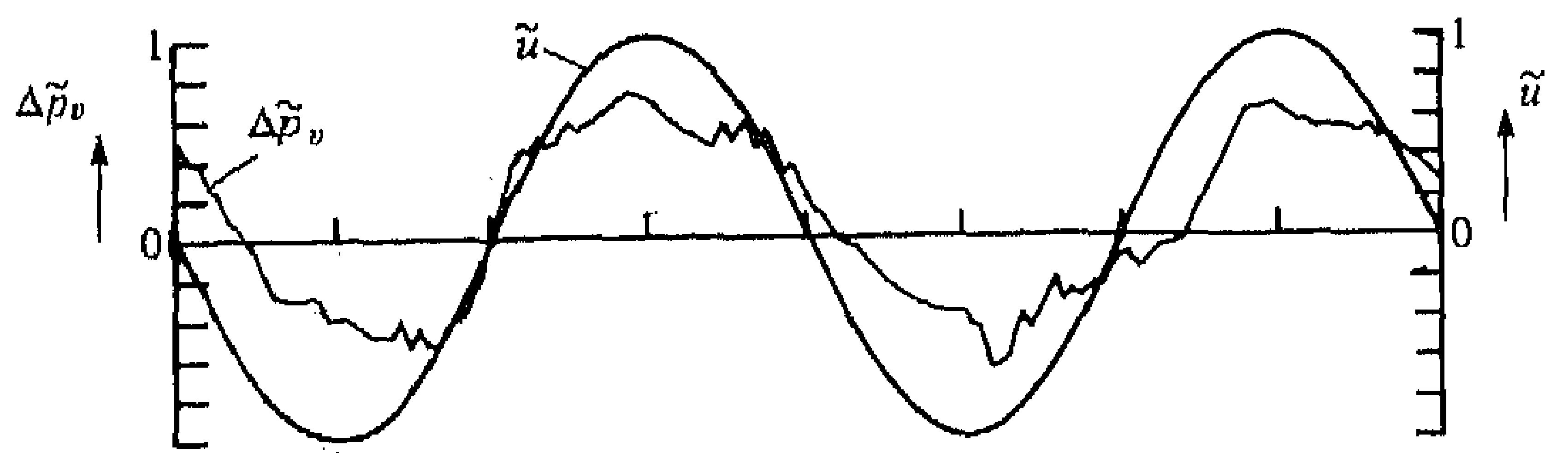

Fraure 18. The scaled pressure $\Delta \tilde{p}_{v}$ as a function of time for $4 \pi<t<8 \pi$.

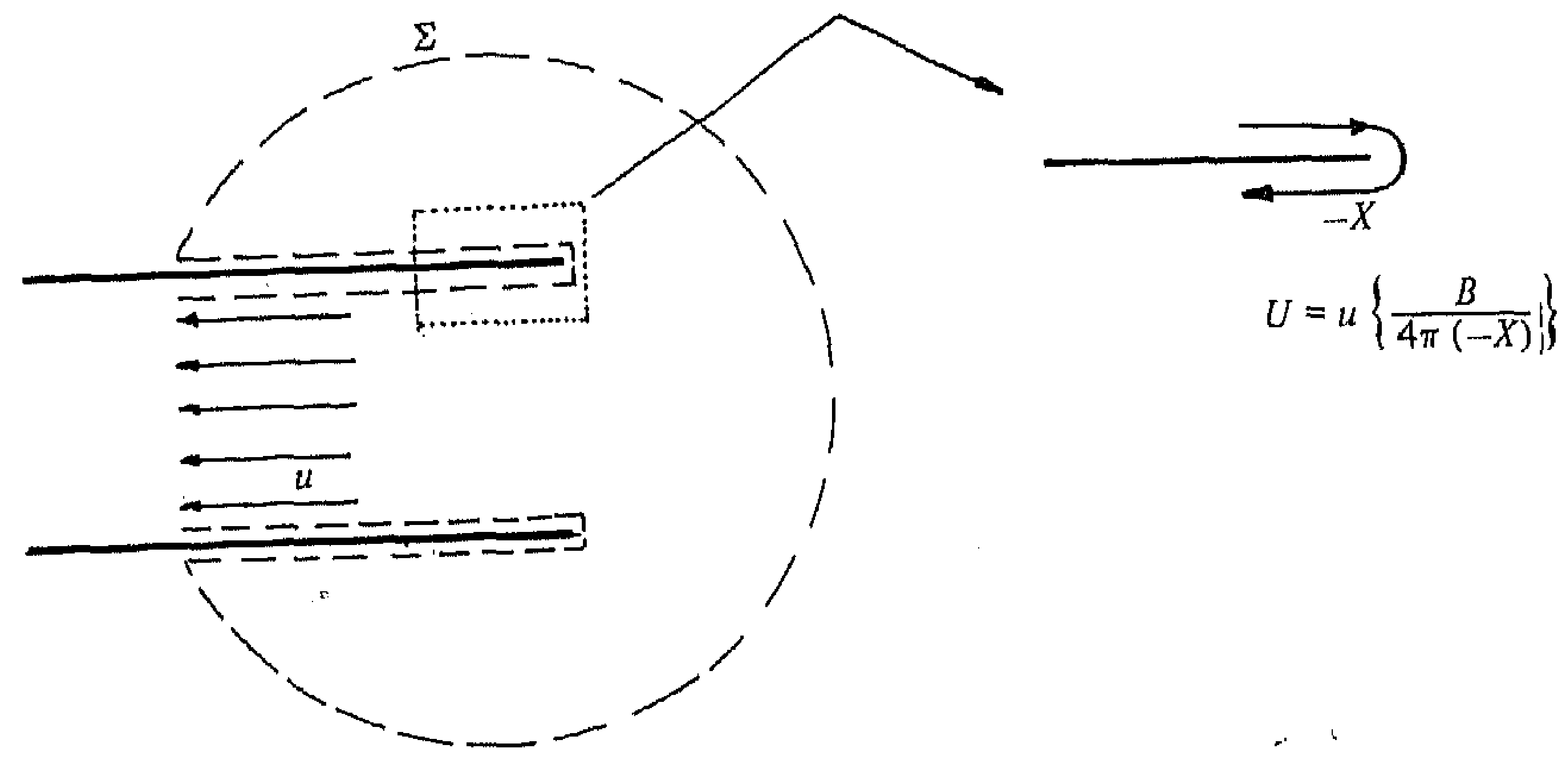

Figure 19. Near the edge of a circular pipe the flow is effectively two-dimensional and can bo compared with the flow between parallel plates if the distance $B$ between the plates is replaced by the tube radius $R$, as follows from a momentum balance of the fluid contained in $\Sigma$.

already nearly periodic and the numerical results shown in figure 18 are during the third and fourth cycle for $N_{0}=20$. The results are obtained for $N_{0}=5,15,17,20$. It follows from these results that $\Delta \tilde{p}_{v}$ is almost exactly in phase with $\tilde{u}$. The relation between $\Delta \tilde{p}$ and $\tilde{u}$ appears to be given by $\Delta \tilde{p}_{v}=0.6 \tilde{u}$, or, using (6.21),

$$
\Delta p_{v}=0 \cdot 6 \rho \Omega B(\widehat{u} / \Omega B)^{\frac{2}{3}} u, \dagger
$$

the velocity at the exit being given by

$$
u=\hat{u} \sin \Omega t .
$$

Relation (7.1) results from vortex formation and shedding near the edge of equh of the plates in figure 14 and drawn again in figure 19, when the velocity far from the edges is $\hat{u} \sin \Omega t$. Since this vortex formation takes place in a small region near the edge, inclicated schematically in figure 19 , we can apply (7.1) equally well to vortex formation and shedding at the edge of a cylindrical tube, provided we use the appropriate velocity near its edge as a base. To find this we note that in the channel the complex conjugate velocity is, from (6.7),

$$
\frac{d w}{d z}=\frac{u}{\zeta}
$$

$\uparrow$ In Disselhorst (1978) a numerical factor of 0.8 is used instead of 0.0 . This was based on the average of the results for $N_{0}=5,15,17$ and 20. If we weight the rosults for $N_{0}=5$, which means a very small number of vortices, less heavily and let those for $N_{0}=15,17$ and 20 mainly determine the result, the relation (7.1) represents the computed results botiter. 
when, far from the edges between the plates, the velocity is $u$ in the $x$ direction. From the mapping relation (5.2) it follows that near $z=i B$ we bave

$$
Z=z-i B \sim-\frac{B}{4 \pi} \zeta^{2},
$$

whence, in the neighbourhood of the edge of the upper plate in figure 14,

$$
w \sim W=u\left\{\frac{B}{\pi}(-Z)\right\}^{\frac{1}{2}} .
$$

The velocity in the $x$ direction is, with $Z=X+i Y$, near the edge in the channel

$$
U=u\{B / 4 \pi(-X)\}^{\frac{1}{2}} .
$$

In analogy with (7.6) for a channel we write, for the velocity in a circular pipe in the vicinity of the edge,

$$
U=u\{q R / 4 \pi(-X)\}^{\frac{1}{2}},
$$

$-X$ being the distance along the pipe from the edge. In (7.7) $q$ is a constant to be determined. Potential flow theory gives that with a potential $c Z^{\frac{1}{2}}$ for the twodimensional flow around the edge $Z=0$ of a plate is associated a suction force

$$
F=\frac{1}{4} \pi \rho c^{2}
$$

(Batchelor 1967, p. 412). Hence associated with the potential $W$ in (7.5) is a suction force $\frac{1}{4} \rho_{0} u^{2} B$ per unit width of the plate. For a circular pipe a momentum balance of the fluid enclosed by the surface $\Sigma$ indicated in figure 19 gives for the suction force

or

$$
2 \pi R F=\frac{1}{2} \rho u^{2} \pi R^{2}
$$

$$
F=\frac{1}{4} \rho u^{2} R \text {. }
$$

Then, if the potential in the vicinity of the edge is represented, according to $(7.5)$ and (7.7), by

$$
W=u\left\{\frac{q R}{\pi}(-Z)\right\}^{\frac{1}{2}},
$$

it follows from (7.8) and (7.9) that $c^{2}=u^{2} R / \pi$, and accordingly $q=1$. Hence the expression for $\Delta p_{v}$ valid for a pipe is obtained from (7.1) by replacing $B$ by $R$ :

$$
\Delta p_{v}=p-p_{0}=0.6 \rho_{0} \Omega R(\hat{u} / \Omega R)^{\frac{2}{3}} u .
$$

This relation is the main theoretical result of the present study and provides, for high Strouhal numbers, a relation between the excess pressure, as caused by vortex formation, and the velocity at the mouth of the pipe.

The validity of (7.11) might be verified by measuring both $p-p_{0}$ and $u$ at the open end. However, at the low values of $\imath$ for which (7.11) may be expected to hold, pressure measurements are very difficult to perform. In order to verify whether (7.11) may be used to predict the amplitude of standing waves, we proceeded as follows. Using the symbols $P_{w}, P_{\text {uc }}$ and $P_{p}$ introduced in (2.4), (2.6) and (2.8), representing respectively boundary layer dissipation, radiated acoustic power and work performed on the gas in the pipe by the piston, and in addition denoting the mean value of $\Delta p_{v} u$ with $P_{\text {end }}$, the mean energy balonce can be written as

$$
P_{p}-P_{\text {end }}=P_{w}+P_{\mathrm{ac}}
$$




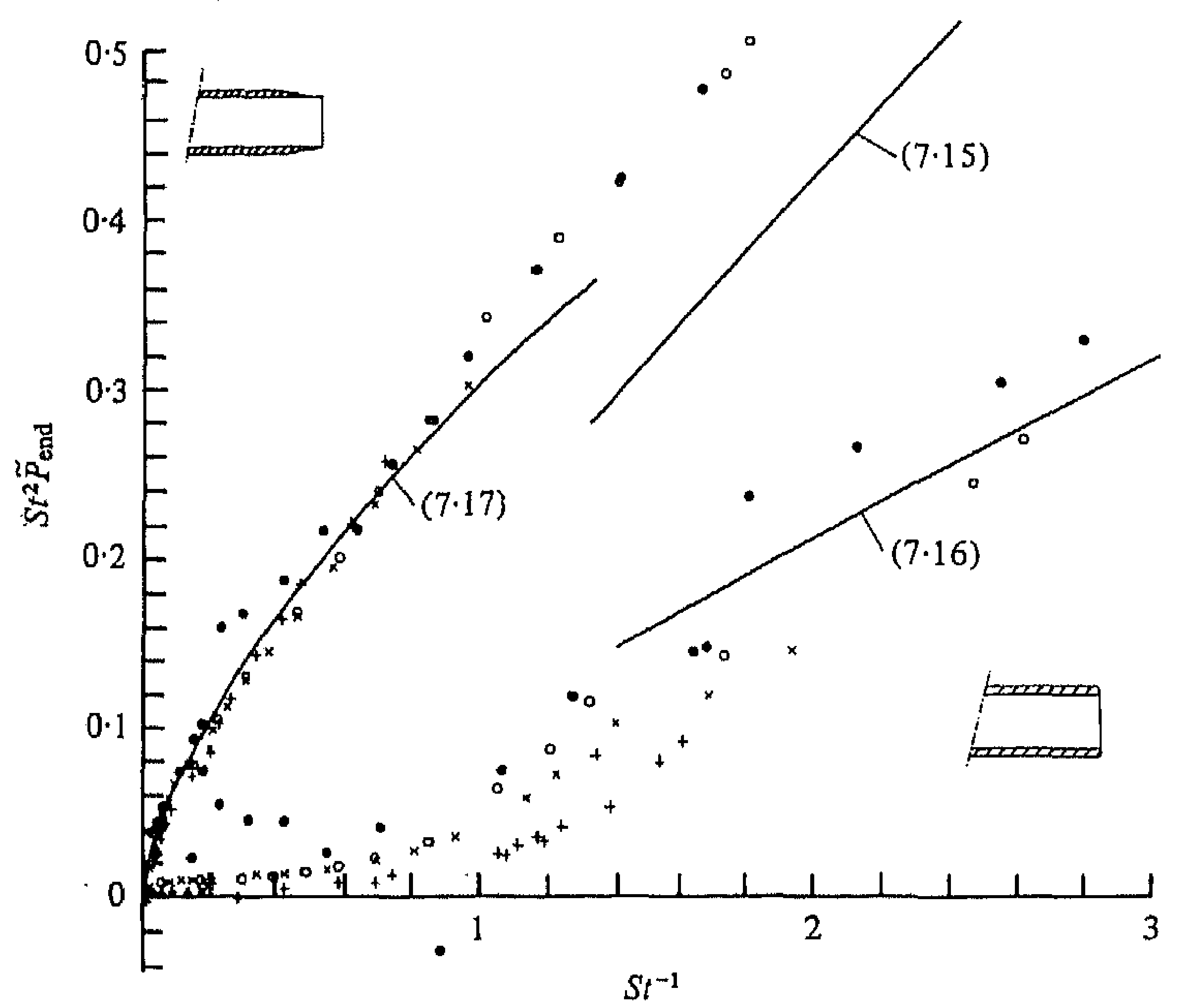

FraURe 20. The dimensionless loss at the open end of a tube as a function of the Strouhal number Solid lines, theoretical results.,$+ L=1.1 \mathrm{~m} ; x, L=1.8 \mathrm{~m} ; O, L=3.3 \mathrm{~m} ; 0, L=6.3 \mathrm{~m}$.

We render $P$ dimensionless by writing

$$
\widetilde{P}=P / \pi \rho_{0} \Omega^{3} R^{5} .
$$

Then, from $(2.3),(2.4),(2.6),(2.8),(7.12)$ and $(7.13)$, it follows that in dimensionless form the energy balance reads

$$
S t^{2} \tilde{P}_{\text {end }}=\left\{2 \frac{\hat{p}_{p}-p_{0}}{\rho_{0} a_{0} \Omega \delta} K\right\}^{-1}-\frac{\pi}{2^{\frac{5}{2}}} \frac{S h}{K}\left(1+\frac{\gamma-1}{\operatorname{Pr}^{\frac{1}{2}}}\right)-\frac{1}{8} \kappa K .
$$

The quantity $\left(\hat{p}_{p}-p_{0}\right) / \rho_{0} a_{0} \Omega \delta$ was defined as $Q$ in $(2.10)$ in the course of the discussion of waves in pipes with a round edge. In that case there is at high Strouhal numbers no vortex formation at the mouth and consequentily $\widetilde{P}_{\text {end }}$ tends to zero for $S t \gg 1$ and round edges. Indeed, the relation (2.11) is recovered from (7.14) when we put the right-hand side equal to zero. For all the experiments in which $Q$ was measured (see figure 3) $S t^{2} \widetilde{P}_{\text {end }}$ was determined by inserting $Q$ in (7.14). The results have been plotted in figure 20 against $S t^{-1}$, both for sharp-edged and smooth-edged pipes of various lengths. Bearing in mind that all experimental observations apply to resonance, it follows that with each value of the pipe length $L$ a different value of $S h$ and of $K$ is connected. For sharp edges, however, it appears from the results in figure 20 that the relative influence of $K$ and $S h$ is only slight. For round edges the influence is somewhat larger. This follows also from (7.14) because if we insert typical values obtained from figure 3 into (7.14) it appears that with sharp edges the terms with $S h / K$ and with $\kappa K$ in (7.14) are significantly smaller than the remaining ones, whereas with round edges the terms on the right-hand side of (7.14) are of the same order of magnitude. This explains why with sharp edges the Strouhal number almost completely determines the flow. 
We compare the experimental results for $\tilde{P}_{\text {end }}$, as shown in figure 20 , with the available theoretical predictions. For low Strouhal numbers $S t \ll 1$,

$$
\begin{aligned}
& S t^{2} \widetilde{P}_{\text {end }} \sim \frac{2}{3 \pi} S t^{-1} \text { for the sharp edge, } \\
& S t^{2} \widetilde{P}_{\text {end }} \sim \frac{1}{3 \pi} S t^{-1} \text { for the round edge. }
\end{aligned}
$$

These relations are readily obtained from the boundary conditions for quasi-steady flow formulated in (2.14) and (2.15) for smooth edges and (2.16) for sharp edges, therein writing $u=\hat{u} \sin \Omega t$ for $u$. These lines are drawn in figure 20. It follows that the experimentally obtained values for $\tilde{P}_{\text {end }}$ tend to be along these lines, although probably in our experiments the Strouhal numbers were not small enough to fulfil the conditions underlying (7.15) and (7.16).

We are in particular interested in the limit of high Strouhal numbers where we derived the result (7.11) for sharp edges. Using this at the open end of the pipe for the pressure difference above the ambient atmosphere in calculating the mean value of $\left(p-p_{0}\right) u$ at the open end, we obtain, with a view to (7.13),

$$
S t^{2} \tilde{P}_{\text {end }}=0 \cdot 3 S t^{-\frac{2}{3}} \text { for } S t \gg 1 \text { and the sharp edge. }
$$

This relation is drawn also in figure 20 and it is seen that the experimental points are situated very close to the curve predicted by (7.17). One can conclude that down to $S t \sim 1$ the relation (7.4), or in dimensionless form (7.17), predicts the amplitude of the standing acoustic wave very well. In view of the assumptions made in the mathematical model, in particular the assumption [cf. (6.22)] that the vortices are not far from the edge, this is a surprisingly broad range of validity. Finally, for smooth edges we infer from the assumption that no vortex formation or separation takes place at high $S t$, that

$$
S t^{2} \tilde{P}_{\text {end }} \rightarrow 0 \text { for } S t \gg 1 \text { and the round edge. }
$$

This also is very well confirmed by the experiments.

In order to illustrate the results in another way, we have prepared from the experimental data and from the known expressions (2.4) and (2.6) for $P_{\mathrm{ac}}$ and $P_{w}$ the figures $21(a, b)$, which represent in an overall way the relative importance of wall dissipation, acoustic radiation and end losses. $\dagger$ At the constant value 0.0031 of the quantity $\left(\nu / a_{0} R\right)^{\frac{1}{2}}\left\{1+(\gamma-1) / P r^{\frac{1}{2}}\right\}$, these figures illustrate how an $L / R-\delta / R$ plane can be divided into regions where one of these mechanisms dominates. In preparing these plots analytic curves fitting the data in figure 20 have been used.

\section{Comparison with other work}

Relation (7.11) has been found here for a range of parameters in which vortex formation at the open end dominates. We briefly discuss some other boundary conditions which have been proposed in addition to those mentioned in previous sections. Jimenez (1973) and Seymour \& Mortell (1973) assume at the end section that the relation

$$
p-p_{0}=-j u
$$

exists between the excess pressure and the velocity. The outcome of the analysis is

$\dagger$ We are indebted to one of the referees for suggesting that we draw up such a plot. 


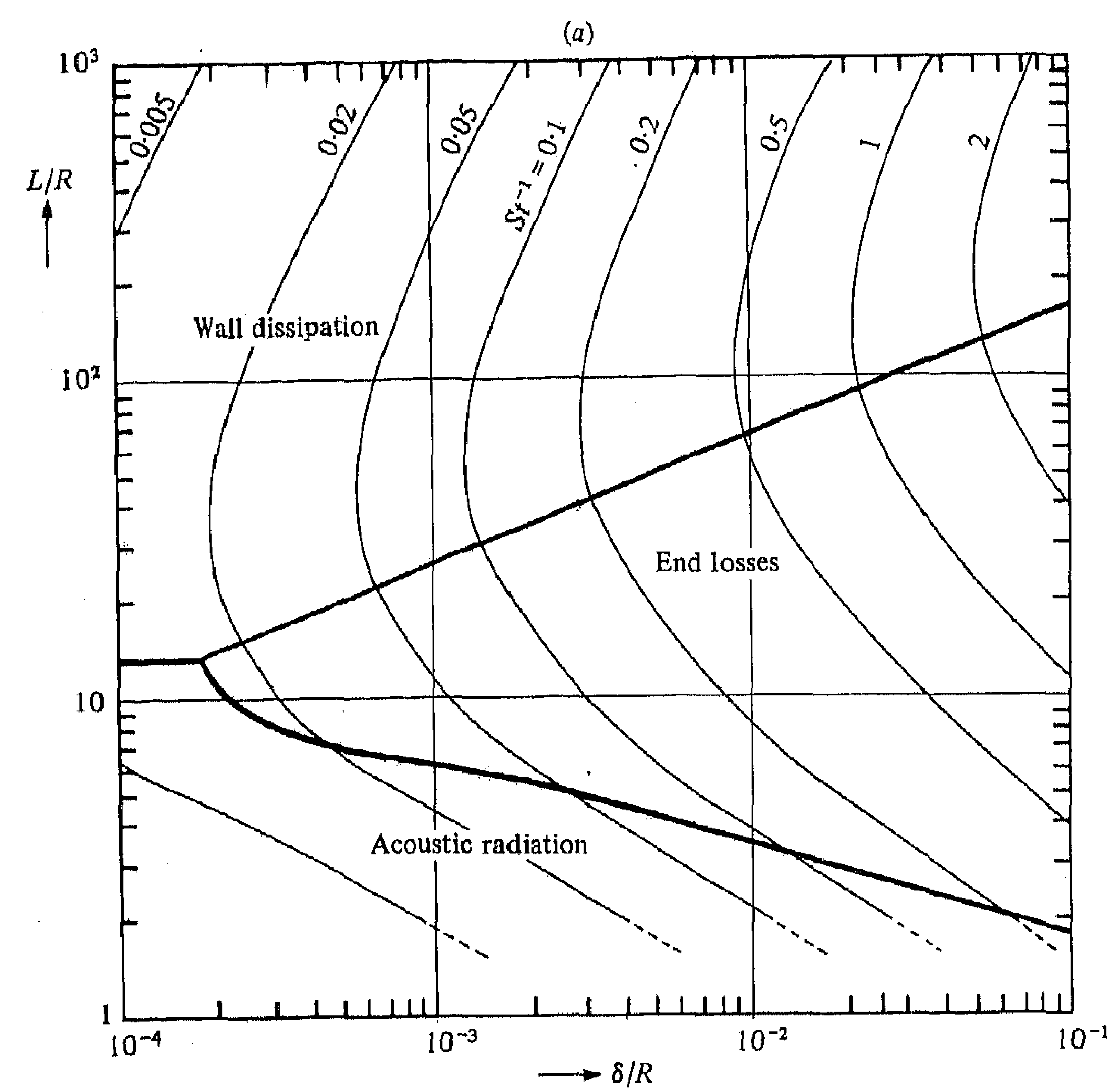

Figure $21(a)$. For legend see page 317.

that, if radiation losses as expressed by (8.1) dominate, $j \sim \hat{u}^{2}$ and $\hat{u} \sim \delta^{\frac{1}{3}}$. In experiments no support for the assumption (8.1) has been found. Nonlinear distortion has been found to lead to formation of shock waves (Lettau 1939; Sturtevant 1974) but then the energy-consuming mechanism is dissipation in the shock wave and not radiation.

As a generalization of (2.16) Sturtevant \& Keller (1978) subjected, in their analysis, the motion in an open pipe to the end condition

$$
\begin{aligned}
& p-p_{0}=-\frac{1}{2} \rho_{0} u^{2}\left(1+k_{\text {in }}\right) \quad \text { at inflow, } \\
& p-p_{0}=-\frac{1}{2} \rho_{0} u^{2}\left(1+k_{\text {out }}\right) \quad \text { at outflow. }
\end{aligned}
$$

Introduction of experimentally obtained data for the pressure at the piston in analytically obtained results, gave a relation between $k_{\text {in }}$ and $k_{\text {out }}$, both for round and for sharp edges. With round edges they found only small deviations from Bernoulli's law, $k_{i_{n}}$ and $k_{\text {out }}$ less than unity, confirming our findings for round edges. For sharp edges they found values for $k_{\mathrm{in}}$ and $k_{\mathrm{out}}$ of 3 and larger. They envisaged vortex generation as a possible cause for these large losses but rejected this on the basis of an order-of-magnitude argument leading to a too small value, 0.2 , of $k_{\text {out }}$. In this argument one ring vortex is supposed to be generated during the part of a cycle in which fluid is ejected from the open end. Between the circulation, $\widetilde{\Gamma}$ say, and $u$ they take the relation

$$
\widetilde{\Gamma} \sim u R
$$


(b)

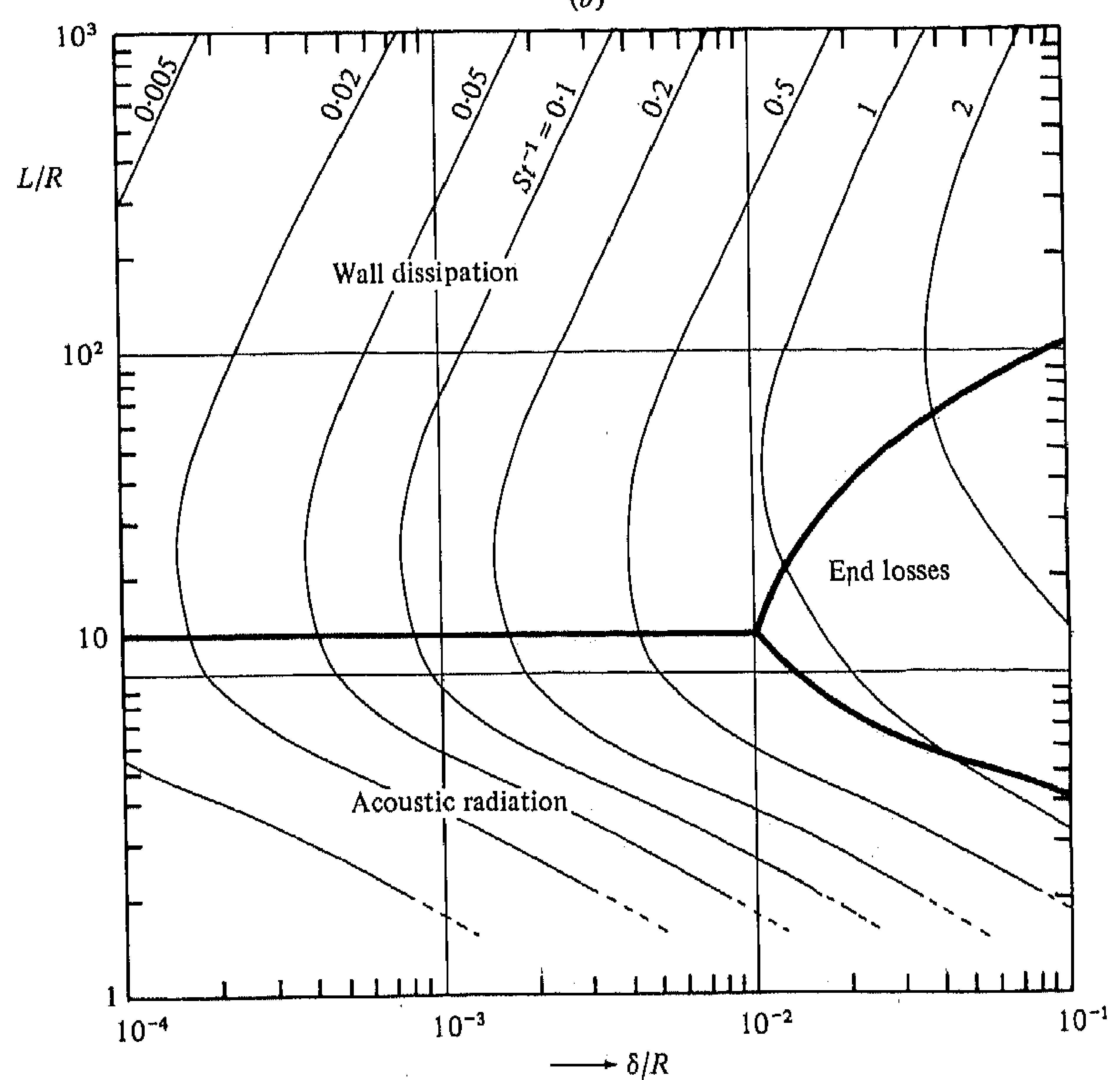

FIGURe 21. Division of the $\delta-L$ plane into three regions in which wall dissipation, end losses or acoustic radiation is the most important mechanism at resonance; $\left(\nu / a_{0} R\right)^{\frac{1}{2}}\left\{1+(\gamma-1) / P r^{\frac{1}{2}}\right\}$ $=0.0031 .(a)$ Sharp edge; $(b)$ round edge.

based on data for a flow accelerated impulsively to velocity $u$. From our experiments it appears that during outflow two vortex rings rather than one are formed. Apart from this, it may be questioned whether (8.3) holds for periodic flow. If we employ the 'slug model' found to be useful (Maxworthy 1977) in similar circumstances, then

$$
\widetilde{\Gamma}=\frac{1}{2} \int u d l,
$$

$l$ being the instantaneous length of the ejected slug of fluid. Thus we find for $u=\hat{u} \sin \Omega t$ that

$$
\widetilde{\Gamma}=\frac{1}{2} \widehat{u}^{2} \Omega^{-1},
$$

and, using this instead of (8.3) in the calculation by Sturtevant \& Keller (1978) in which the $k_{\text {oul }}$ th fraction of the ejected energy is concentrated in a vortex ring, we find

$$
k_{\text {out }} \sim 1.5 S t^{-1}
$$

In the experimental example, used by Sturtevant \& Keller, $S t^{-1}=2 \cdot 48$, and $k_{\text {out }}$ is therefore, according to (8.6), much larger than 0.2 . Also we can, assuming the end loss $(2 \pi / \Omega) P_{\text {end }}\left[P_{\text {end }}\right.$ defined in (7.12)] in a period of the motion to be equal to the energy in a vortex ring, ask how according to $(7.11) \tilde{\Gamma}$ is related to $\hat{\imath}$. We obtain

$$
\widetilde{\Gamma} \sim \Omega R^{2}(\hat{u} / \Omega R)^{\frac{4}{3}},
$$


which differs considerably from (8.3). The present authors are therefore not convinced by the argument in Sturtevant \& Keller (1978) to the effect that end losses cannot be attributed to vortex formation. In fact our present work shows that in a certain range of parameters the generation and rolling-up of vortex sheets does explain observed end losses.

\section{Conclusion}

In this paper we have considered energy-consuming mechanisms in resonant oscillations in open acoustic tubes. The power loss by boundary-layer separation or vortex formation appears to depend strongly on the exit geometry. We have investigated in particular the case of sharp edges at small wave amplitudes or, equivalently, relatively high Strouhal numbers. As visualized in figures 12 and 13 an important mechanism is the generation and shedding of vortices. This process has been simulated numerically, which leads to a prediction [equation (7.11)] of the pressure excess at the open end and therefore enables prediction [equation (7.17)] of the power loss. The agreement with experimental results turns out to be very satisfactory, both in regard to the power loss and also for the structure of the shed vortices. In the case of round edges it is found both from analysis and experiment that viscous and thermal dissipation, and to a lesser extent acoustic radiation, determine the flow at high Strouhal number. At large amplitudes, which means low Strouhal numbers, the amplitude can be fairly well predicted by adopting the model of jet formation at outflow and sinklike flow at inflow. The experimental results show that in particular the boundary condition proposed in Van Wijngaarden (1968) as a result of this model, gives a good prediction of the power loss at the open end. Finally it should be noted that it remains desirable to extend the numerical calculations to moderate amplitudes of the wave motion, for which the assumption $\zeta \sim \frac{1}{2} z^{2}$ [cf. (6.22)] is no longer valid. The success obtained here for high Strouhal numbers with the model developed in $\$ 5$ makes it likely that in that way a better prediction than (7.11) may be obtained when $S t^{-1}$ is of order one and larger.

\section{REFERENCES}

Batohelon, G. IK. 1967 An Introduction to Fluid Dynamics. Cambridge University Press.

Chester, W. 1964 Resonant oscillations in closed tubes. J. Fluid Mech. 18, 44.

CHU, B. T. \& YING, S. J. 1963 Thermally driven nonlinear oscillations in a pipe with travelling shock waves. Phys. Fluids 6, 11.

Clements, R. R. \& MAUli, D. J. 1976 The representation of sheets of vorticity by discreto vortices. Prog. Aero. Sci. 16, 129.

CrUIKsHaNK, D. B. 1972 Experimental investigations of finite amplitude acoustic oscillations in a closed tube. $J$. Acoust. Soc. Am. 52, 1024.

Dissecfonst, J. H. M. 1.978 Acoustic resonance in open tubes. Ph.D. thesis, Twente Univorsity of Technology, Enschede.

Disseminorst, J. H. M. \& VAN WIJNGAARDEN, L. 1979 Oscillatory flow around the elge of a flat plate. J. Engng Malh. 13, 271.

Jimmanz, J. 1973 Nonlinear gas oscillations in pipes. Part 1. Theory. J. Fluid Mech. 59, 23.

LeTrau, E. 1939 Messungen an Gasschwingungen grosser Amplitudo in Rohrleitungon. Diseh. Kraftfahrtforsch. 39, 1. 
Levine, H. \& Schwinger, J. 1948 On the radiation of sound from an unflanged pipe. Phys. Rev. 73, 383 .

Liahthrus, M. J. 1978 Waves in Fluids. Cambridge University Press.

Maxworthy, T. 1977 Some experimental studies on vortex rings. J. Fluid Mech. 81, 465.

Merri, P. 1973 Theoretische und experimentelle thermoakustische Untersuchungen am Kolbengetriebenen Resonanzrohr. Ph.D. thesis, Eidgenössische Technische Hochschule Zürich.

Prandte, L. 1961 Collected Works, vol. II., pp. 703-704. Springer.

RAYLEIGH, J. W. S. 1945 The Theory of Sound, cha. 19. Dover.

SaFfman, P. G. \& Baker, G. R. 1979 Ann. Rev. Fluid Mech. 11, 95.

Seymour, B. R. \& Morteds, M. P. 1973 Nonlinear resonant oscillations in open tubes. J. Fluid Mech. 60, 733.

Sturtevant, B. 1974 Nonlinear gas oscillations in pipes. Part 2. Experiments. J. Fluid Mech. $63,97$.

Sturtervant, B. \& Keller, J. J. 1978 Subharmonic nonlinear resonances in open tubes. Part II : Experimental investigation of the open-end boundary condition. Z. angew. Math. Phys. 29, 473.

Temmin, S. 1968 Nonlinear gas oscillations in a resonant tube, Phys. Fluids 11, 960.

VAN WrJngaARden, L. 1968 On the oscillations near and at resonance in open pipes. Proc. A.S.C.E., J. Engng Math. 2, 225.

VAN WIJNGAARDEN, L. \& WoRMGOOR, W. 1974 Investigations on resonant acoustic waves in open pipes. In Finite Amplitude Wave Effects in Fluids (ed. L. Bjørnø), p. 75. IPC Science and Technology. 

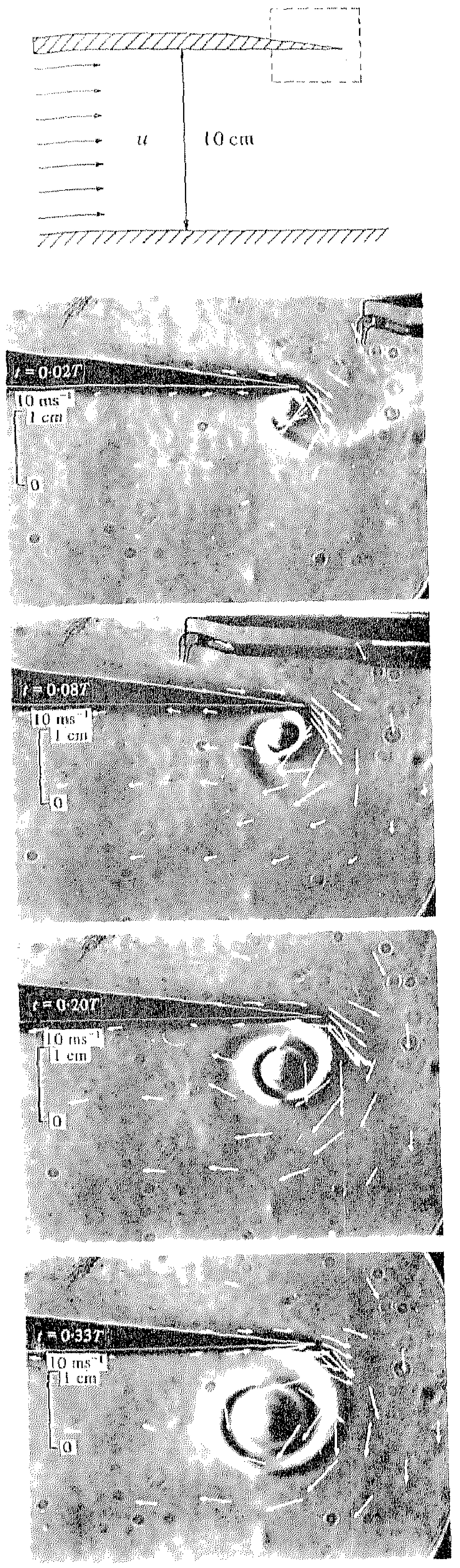

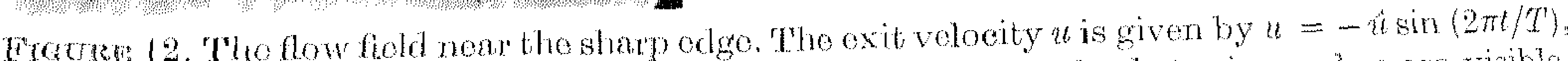
where $\hat{t}=21 \mathrm{~ms}$ and $T=0.035 \mathrm{~s}$. In some of the pictures the hot-wire probes are visible. DISSECHORSE AND WIJNGAARDEN
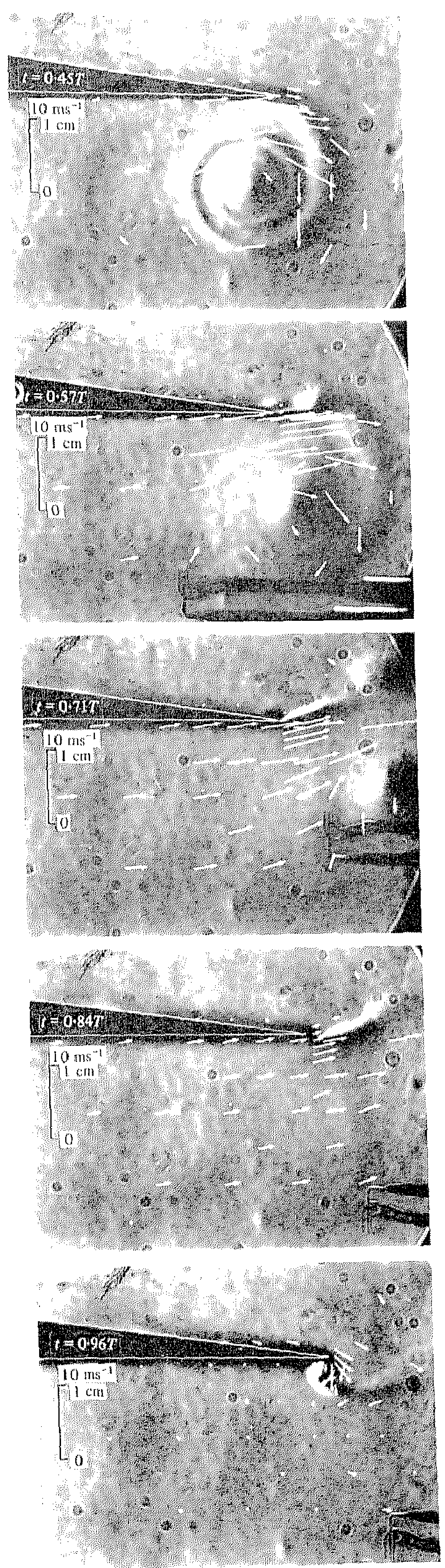

$($ facing p. 320) 


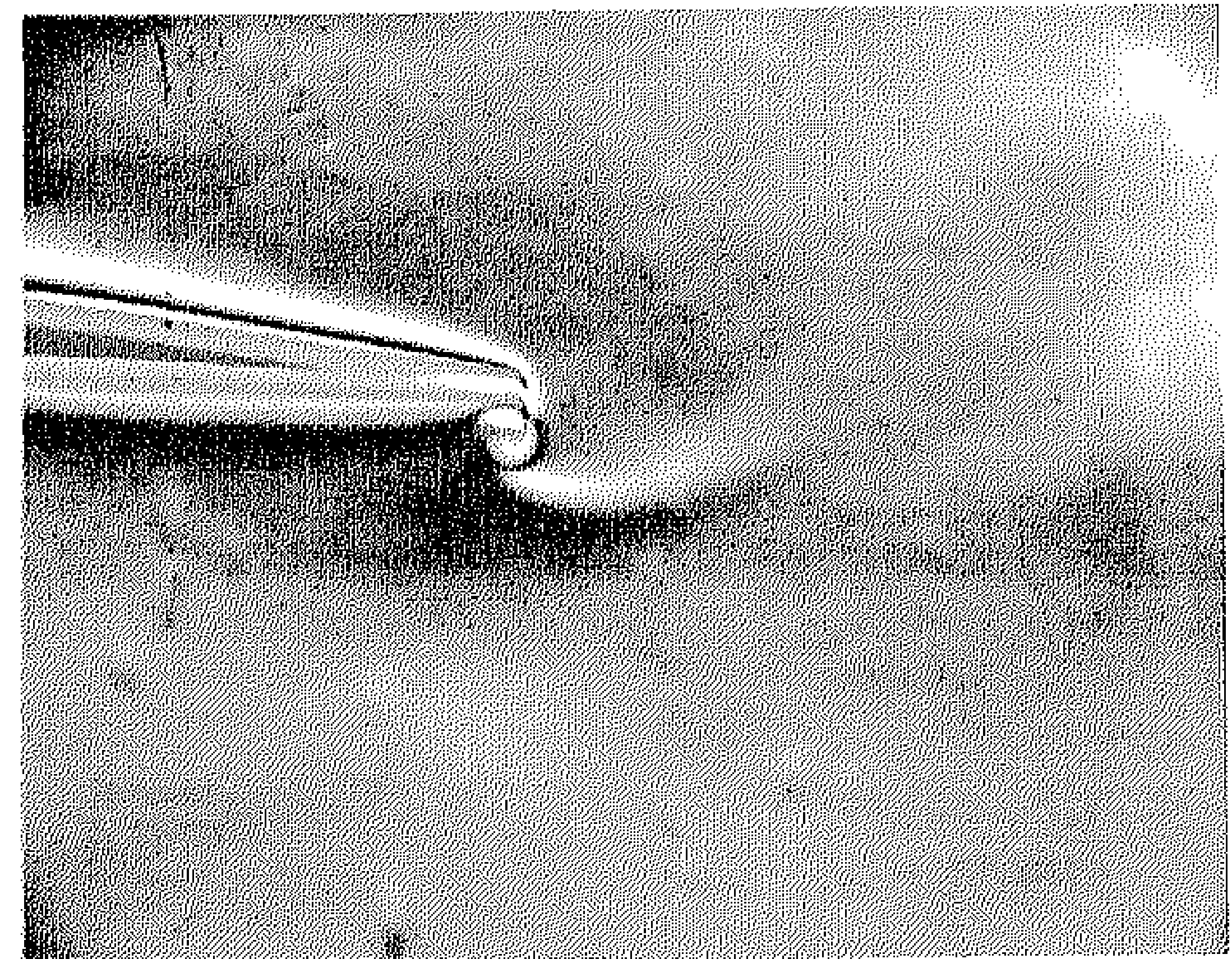

(a)

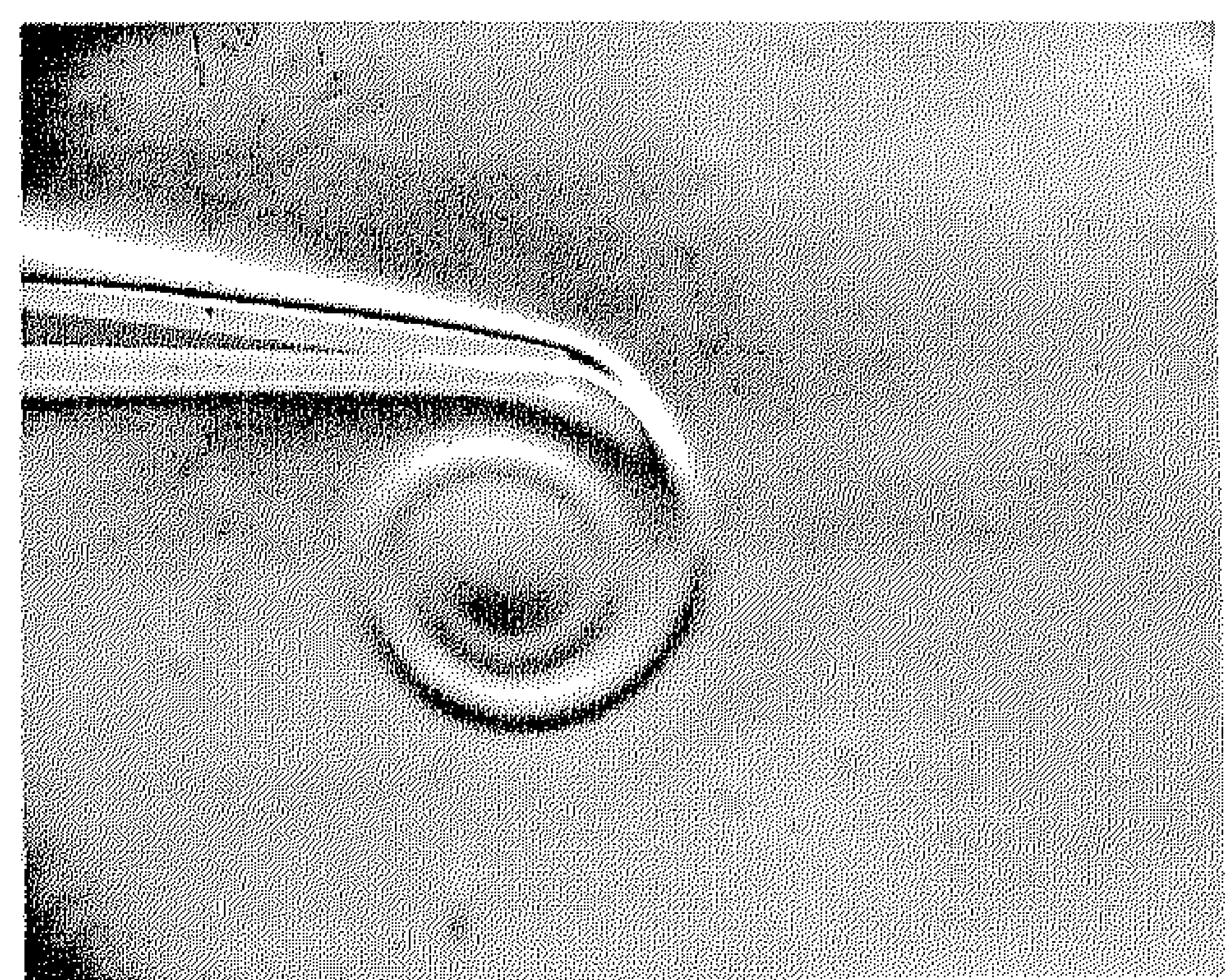

(c)

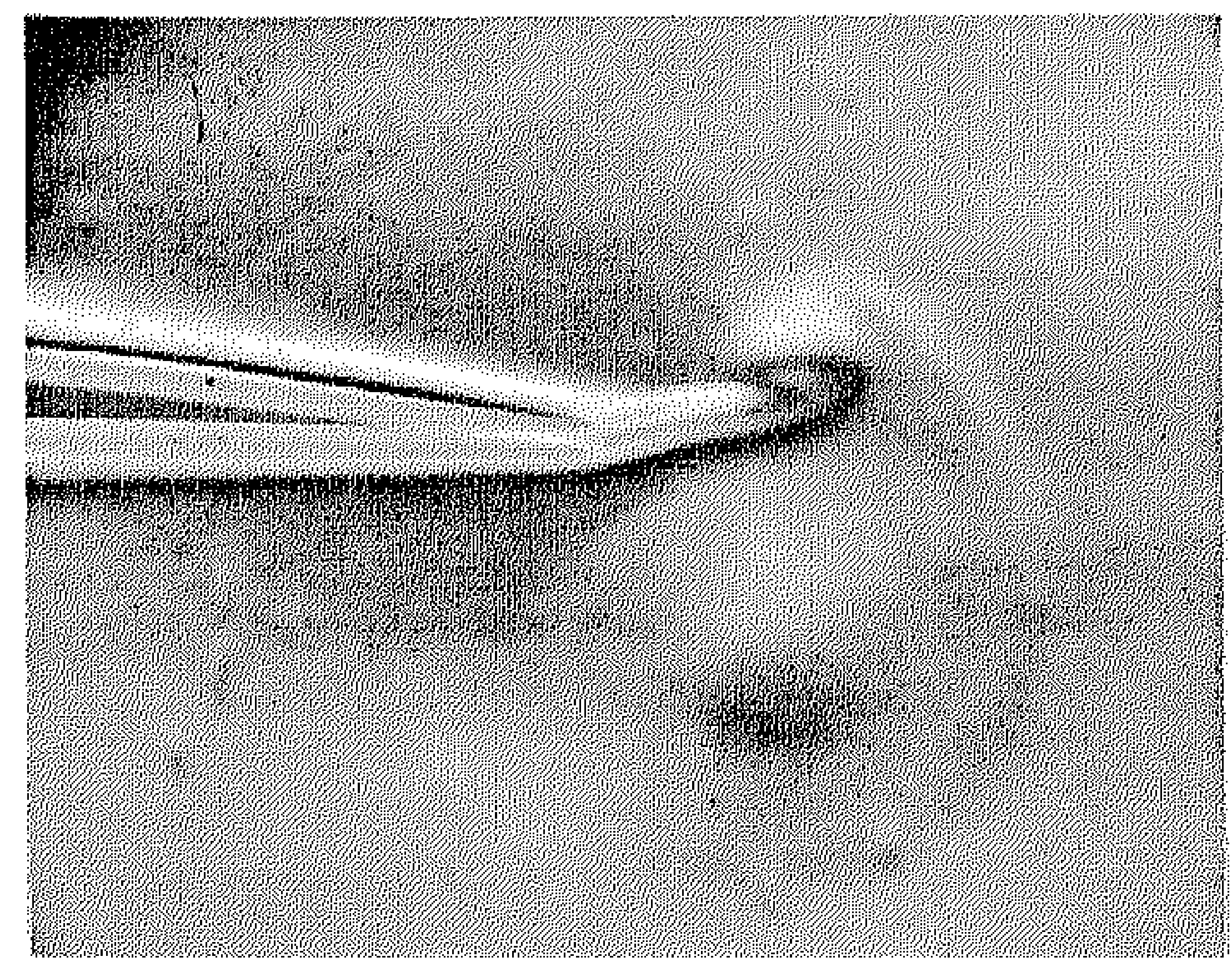

$(e)$

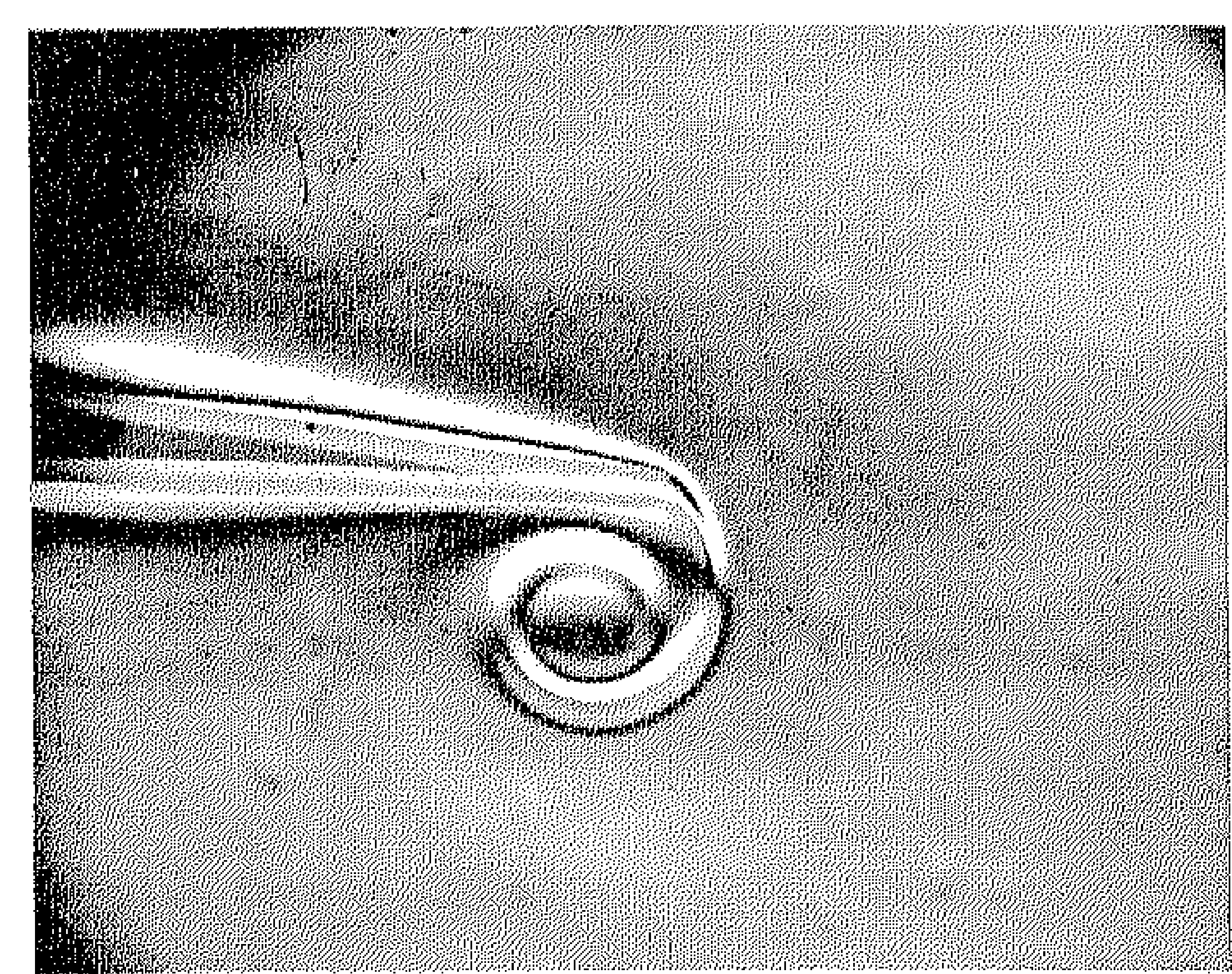

(b)

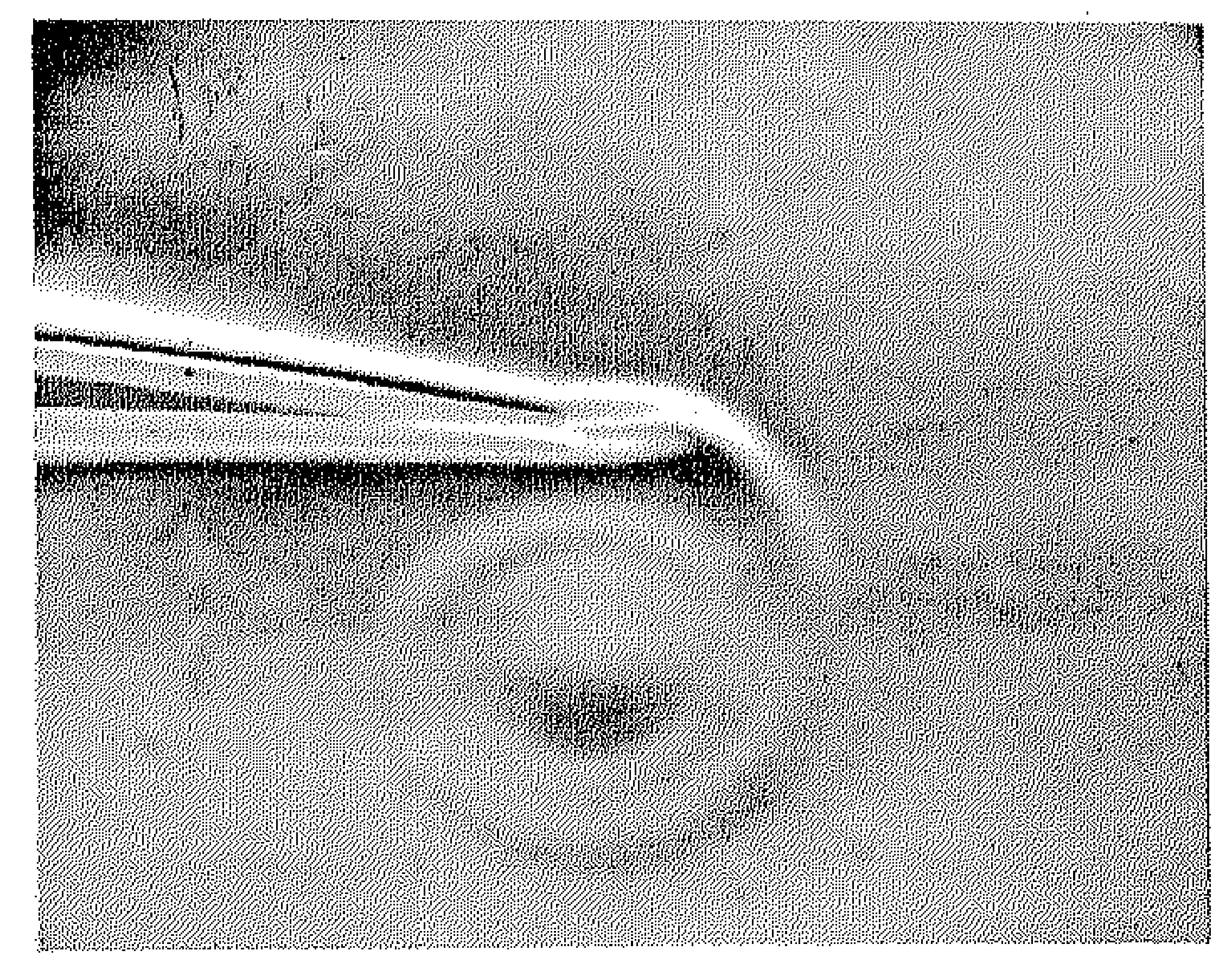

(d)

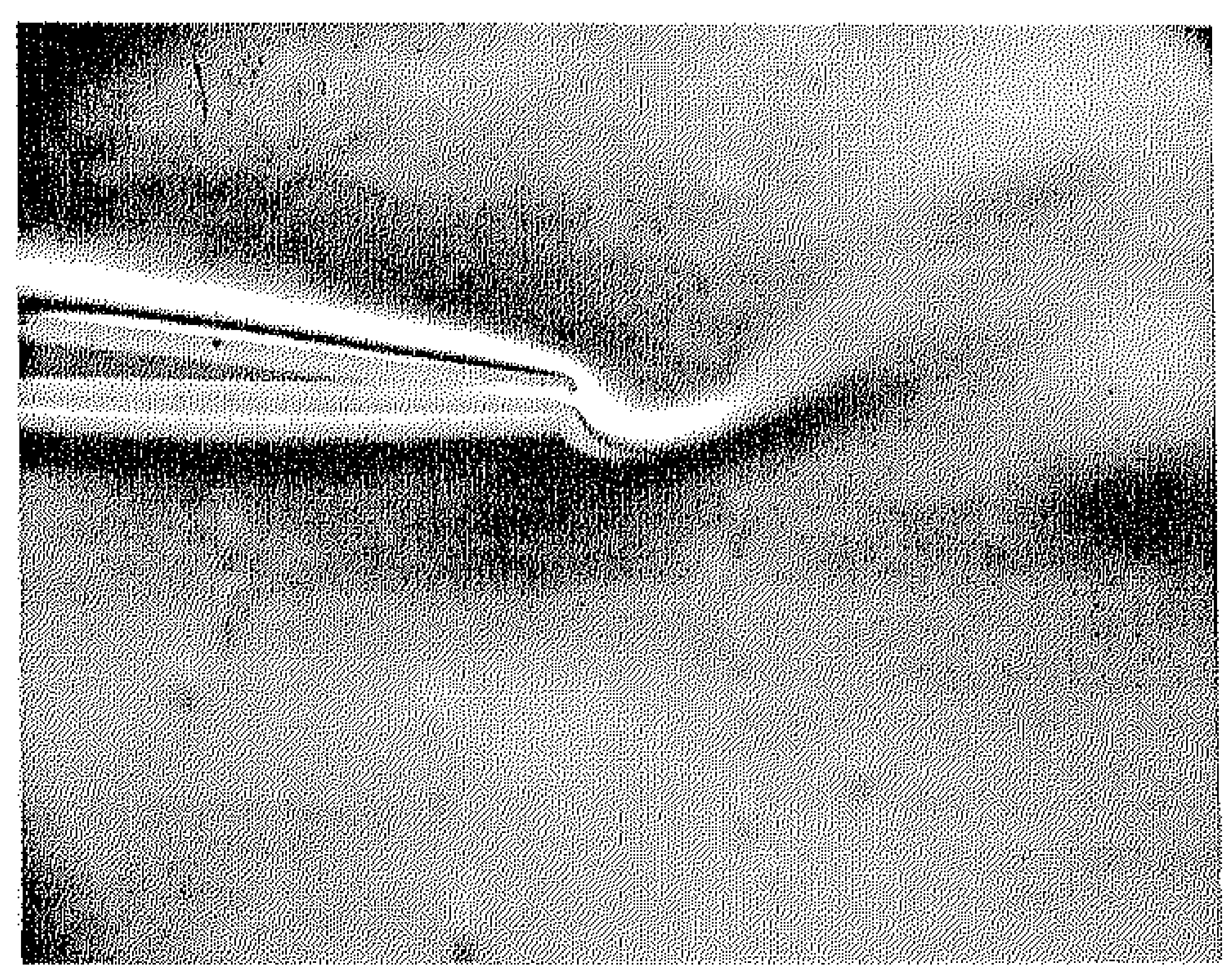

$(f)$

Ficune 13. Flow field near the sharp edge visunlized by the Wollaston method; $S t=21$, the period $T=0.02 \mathrm{~s}$. The pictures $(a)-(f)$ are at $t / T=0,0.25,0.41,0.53 .(1.65 \pi$ and 0.900 . 